\title{
REVIEW
}

\section{The Impact of 7-valent Pneumococcal Conjugate Vaccine on Invasive Pneumococcal Disease: A Literature Review}

Tin Tin Htar Myint · Harish Madhava · Paul Balmer · Dina Christopoulou · Sepideh Attal · Damianos Menegas . Ralf Sprenger · Eric Bonnet

To view enhanced content go to www.advancesintherapy.com Received: December 4, 2012 / Published online: February 7, 2013

(c) The Author(s) 2013. This article is published with open access at Springerlink.com

\section{ABSTRACT}

Introduction: Streptococcus pneumoniae can cause invasive pneumococcal diseases (IPD), such as bacteremic pneumonia, bacteremia, meningitis, and sepsis, and non-IPDs, such as otitis

\section{T. T. H. Myint $(\bowtie) \cdot$ E. Bonnet}

Pfizer ESAT Vaccines, 23-25 Avenue du Docteur Lannelongue, 75014 Paris, France

e-mail: Myint.TinTinHtar@pfizer.com

H. Madhava · P. Balmer · D. Christopoulou Pfizer Ltd., Pfizer Europe, Walton Oaks, Dorking Road, Walton on the Hill, KT20 7NS, UK

S. Attal

Pfizer France, 23-25 Avenue du Docteur Lannelongue, 75014 Paris, France

D. Menegas

Pfizer Hellas AE, 243 Mesoghion Avenue, N. Psychico Athens, GR-15451, Greece

\section{R. Sprenger}

Pfizer Germany, Linkstrasse 10, 10785 Berlin, Germany

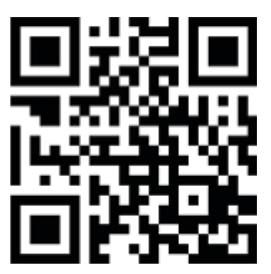

Enhanced content for Advances in Therapy articles is available on the journal web site: www.advancesintherapy.com media, nonbacteremic pneumonia, and upper respiratory tract infections. It was estimated in 2000 that, worldwide, S. pneumoniae was responsible for 826,000 deaths annually in children aged between 1 month and 5 years. A 7-valent pneumococcal conjugate vaccine (PCV7) was licensed in 2000 in the USA and in 2001 in Europe.

Methods: A literature search was performed in PubMed to identify studies assessing the impact of routine childhood PCV7 vaccination on pneumococcal morbidity and mortality. Here, the impact on IPD is reported.

Results: A total of 37 articles reporting impact data on IPD were included in this review: four from Australia, 17 from western Europe, and 16 from North America. In vaccine-eligible children in the postvaccination period, a reduction ranging from $39.9 \%$ in Spain to $99.1 \%$ in the USA in vaccine-type (VT) IPD incidence, compared with the prevaccination period, was reported in 18 studies. All but one of the 30 studies assessing the impact of PCV7 on all-type IPD reported a reduction ranging from $1.7 \%$ in Spain to $76.3 \%$ in Australia. In addition, the majority of studies reported reductions in VT and all-type IPD incidence in age groups that were not vaccine eligible. 
Conclusions: The results from this review illustrate that PCV7 has had a significant impact on IPD across all ages through its use in pediatric immunization programs. With the introduction of 13-valent pneumococcal conjugate vaccine (PCV13) further reductions in the incidence of IPD due to the six additional serotypes included, as well as continued protection against IPD due to PCV7 serotypes may be expected. Robust surveillance systems are essential for the evaluation of the impact of PCV13 on all-type IPD and for monitoring the evolution of non-VT IPD.

Keywords: Direct vaccine impact; Immunology; Indirect vaccine impact; Infectious diseases; Invasive pneumococcal disease; Streptococcus pneumoniae; Vaccine impact; 7-valent pneumococcal conjugate vaccine

\section{INTRODUCTION}

Streptococcus pneumoniae is a Gram-positive diplococci surrounded by a polysaccharide capsule that protects it from the human immune system. More than 90 serotypes have been identified, with a serotype distribution that varies geographically. However, only a limited number of serotypes cause invasive pneumococcal disease (IPD) throughout the world [1]. $S$. pneumoniae causes invasive diseases, such as bacteremic pneumonia, bacteremia, meningitis, and sepsis, and non-IPDs, such as otitis media and upper respiratory tract infections [2]. It was estimated in 2000 that, worldwide, $S$. pneumoniae was responsible for 826,000 deaths annually in children aged between 1 month and 5 years [3]. In 2005, over a 12-month period, 23,470 cases of IPD were reported in 18 European countries, with the incidence varying from 0.4 in Italy to 20.2 and 20.3 per 100,000 general population in Finland and Denmark, respectively [4]. In 1998-1999 in eight geographical areas in the USA, the incidence of IPD ranged from 19.0 to 29.9 per 100,000 general population [5]. However, the surveillance systems, case definitions, and diagnostic standards for IPD in Europe are very heterogeneous making direct comparisons difficult.

A 7-valent pneumococcal conjugate vaccine (PCV7) was licensed in 2001 in Europe and introduced into the national immunization programs (NIPs) as a universal childhood vaccine in around 2006-2008. Its introduction into the NIPs in different countries varied, with some countries initially only recommending vaccination for high-risk children before generalizing to all children (e.g., UK, Germany, and France). PCV7 was licensed and introduced into the NIP in 2000 in the USA. In Australia, it was licensed in 2002 and was initially recommended for high-risk children, but in 2005 PCV7 was introduced into the NIP for all children.

PCV7 has now been in use for over 10 years and many studies have reported its effectiveness and impact [6-9]. PCV7 has been replaced by 13 -valent pneumococcal conjugate vaccine (PCV13) in many countries, and a 10 -valent conjugate pneumococcal vaccine is also available. These higher-valent conjugate vaccines include some of the important emerging serotypes, while continuing to protect against the serotypes in PCV7. In this review, the wealth of data available on the impact of routine childhood PCV7 in reducing alltype and vaccine-type (VT) IPD, in vaccineeligible (direct effect) and nonvaccine-eligible populations (indirect effect) in North America, western Europe, and Australia is summarized.

\section{MATERIALS AND METHODS}

The data presented are part of a larger global literature review that assessed the impact of PCV7 in different pneumococcal disease areas. 
The PubMed search was performed using the following terms: (pneumonia OR "invasive pneumococcal disease" OR IPD OR "otitis media" OR death) AND ([pneumococcal AND conjugate AND vaccin*] OR PCV).

In this paper, the data on the impact of PCV7 vaccination in infants on the incidence of IPD (VT and all type) in the general population is summarized, in vaccine-eligible children (direct effect) and in nonvaccineeligible age groups (indirect effect). Studies that were published between January 2000 and March 2011, and performed in western Europe, North America, and Australia were included. Before/after studies were included if the impact data (percentage change in crude or adjusted incidence rates) were provided or could be calculated. The calculation used was ([incidence prevaccination - incidence postvaccination]/ incidence prevaccination) ${ }^{\star} 100$.

Publications that reported efficacy (i.e., randomized clinical trials), modeling or health economics studies, studies on specific populations (e.g., those with comorbidities, such as HIV positive, patients with sickle cell disease), and studies in specific locations with unknown denominators were excluded.

\section{RESULTS}

A total of 1,007 publications were identified from the global search, and after two rounds of screening 84 were selected for inclusion in the global literature review (Fig. 1). The main reasons for exclusion were: cohort study with no comparative group (epidemiology data); modeling or cost-effectiveness studies; only specific patient subgroups; review articles; and no incidence data or data sufficient for calculating incidence (mainly missing data on denominator). From these, all 37 articles reporting data on the impact of routine

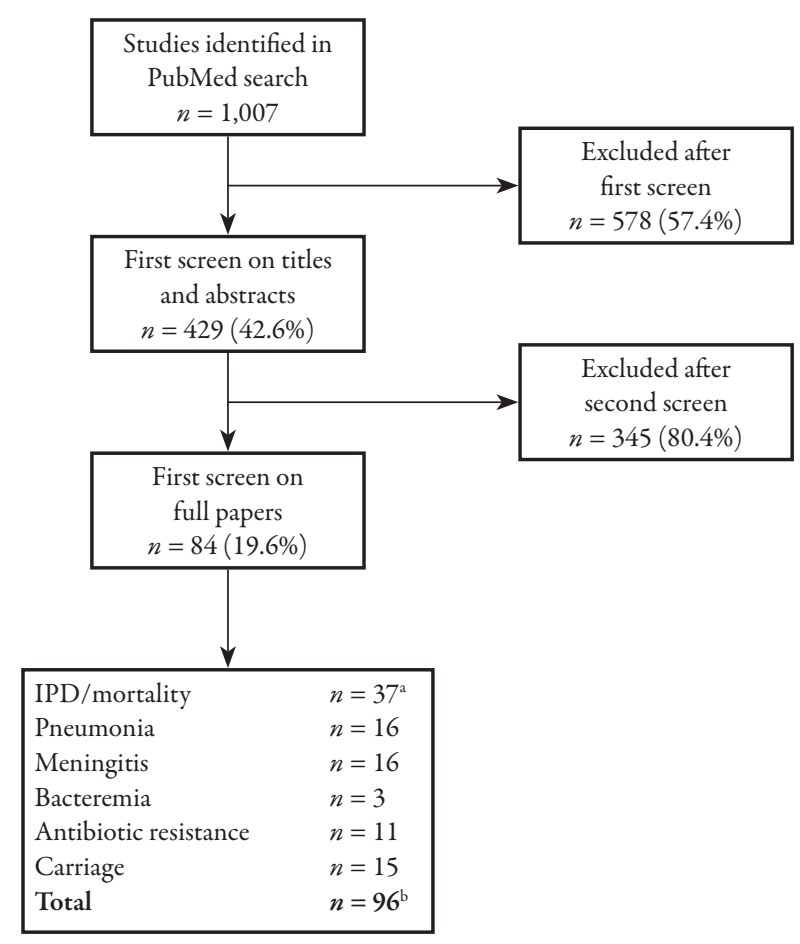

Fig. 1 Flow diagram describing the literature search in PubMed and screening process. ${ }^{a}$ Analyzed in this paper. ${ }^{b}$ Some papers contributed more than one topic. IPD invasive pneumococcal disease

childhood IPD vaccination were analyzed: four from Australia, 17 from western Europe, and 16 from North America [10-46]. The characteristics of these studies are summarized in Table 1 [10-46]. Eight of the European studies were from Spain, two each from Norway and the UK, and one each from Austria, Belgium, Denmark, Germany, and the Netherlands [10-26]. Five of the North American studies used data from the active bacterial core surveillance (ABCs) database; they used the same pre pneumococcal conjugate vaccine (PCV) vaccination period (1998-1999) but analyzed different post-PCV vaccination periods and populations [29-33]. Two other studies from North America used the same database from Alaska; they used the same pre-PCV vaccination period (1995-2000) 
Table 1 Summary of characteristics of included studies (continued on next page)

\begin{tabular}{|c|c|c|c|c|c|}
\hline $\begin{array}{l}\text { Study ID [ref], } \\
\text { country (specific } \\
\text { area) }\end{array}$ & Data sources & $\begin{array}{l}\text { PCV vaccination } \\
\text { policy/schedule }\end{array}$ & $\begin{array}{l}\text { Prevaccination/ } \\
\text { postvaccination time } \\
\text { periods }\end{array}$ & $\begin{array}{l}\text { Age groups } \\
\text { analyzed }\end{array}$ & $\begin{array}{l}\text { Outcomes } \\
\text { analyzed }\end{array}$ \\
\hline $\begin{array}{l}\text { Rendi-Wagner } \\
2009 \text { [10], Austria }\end{array}$ & $\begin{array}{l}\text { National active } \\
\text { hospital and } \\
\text { laboratory } \\
\text { surveillance } \\
\text { database }\end{array}$ & $\begin{array}{l}\text { PCV7 in NIP in } 2004 \\
\text { (reimbursed for at-risk } \\
\text { children) } / 2+1\end{array}$ & $\begin{array}{l}\text { 2001-2004/ } \\
2004-2007\end{array}$ & $\begin{array}{l}<2 \text { years } \\
2-5 \text { years }\end{array}$ & $\begin{array}{l}\text { All-type IPD; } \\
\text { VT IPD; } \\
\text { non-VT IPD; } \\
\text { VRT IPD }\end{array}$ \\
\hline $\begin{array}{l}\text { Hanquet } 2011 \\
{[11], \text { Belgium }}\end{array}$ & $\begin{array}{l}\text { Laboratory } \\
\text { surveillance data } \\
\text { (62\% of hospitals) }\end{array}$ & $\begin{array}{l}\text { PCV7 in NIP in } 2004 \\
\text { - partially reimbursed; } \\
\text { in Jan } 2007 \text { free for } \\
<2 \text { years with catch-up } \\
\text { for } 2 \text { years } / 3+1\end{array}$ & $\begin{array}{l}2002-2003 / \\
2007,2008\end{array}$ & $<5$ years & $\begin{array}{l}\text { All-type IPD; } \\
\text { VT IPD; } \\
\text { non-VT IPD }\end{array}$ \\
\hline $\begin{array}{l}\text { Harboe } 2010 \text { [12], } \\
\text { Denmark }\end{array}$ & $\begin{array}{l}\text { National } \\
\text { laboratory } \\
\text { surveillance } \\
\text { system }\end{array}$ & $\begin{array}{l}\text { PCV7 in NIP in } 2007 \\
\text { with two-dose catch- } \\
\text { up for } 12-17 \text { months/ } \\
2+1\end{array}$ & $2000-2007 / 2008$ & $\begin{array}{l}<2 \text { years } \\
2-4 \text { years } \\
5-17 \text { years } \\
18-49 \text { years } \\
50-64 \text { years } \\
\geq 65 \text { years } \\
\text { Overall }\end{array}$ & All-type IPD \\
\hline $\begin{array}{l}\text { Rückinger } 2009 \\
\text { [13], Germany }\end{array}$ & $\begin{array}{l}\text { National hospital } \\
\text { and laboratory } \\
\text { surveillance } \\
\text { systems }\end{array}$ & $\begin{array}{l}\text { PCV7 in } 2006 \text {, with } \\
\text { catch-up }<2 \text { years } \\
(<5 \text { years for identified } \\
\text { risk groups }) / 3+1\end{array}$ & $\begin{array}{l}1997-2003 / \\
2007-2008\end{array}$ & $\begin{array}{l}<2 \text { years } \\
2-4 \text { years } \\
5-15 \text { years } \\
0-15 \text { years }\end{array}$ & All-type IPD \\
\hline $\begin{array}{l}\text { Rodenburg } 2010 \\
{[14], \text { Netherlands }} \\
\text { (25\% of } \\
\text { population) }\end{array}$ & $\begin{array}{l}\text { National } \\
\text { Reference } \\
\text { Laboratory }\end{array}$ & PCV7 in $2006 / 3+1$ & $\begin{array}{l}2004-2006 \\
2006-2008\end{array}$ & $\begin{array}{l}<2 \text { years } \\
2-4 \text { years } \\
5-49 \text { years } \\
50-64 \text { years } \\
\geq 65 \text { years } \\
\text { Overall }\end{array}$ & $\begin{array}{l}\text { All-type IPD; } \\
\text { VT IPD; } \\
\text { non-VT IPD }\end{array}$ \\
\hline $\begin{array}{l}\text { Vestrheim } 2008 \\
\text { [15], Norway }\end{array}$ & $\begin{array}{l}\text { National } \\
\text { Surveillance } \\
\text { System for } \\
\text { Communicable } \\
\text { Diseases }\end{array}$ & $\begin{array}{l}\text { PCV7 in NIP in } 2006 \\
\text { with catch-up (for } \\
\text { children } 3-6 \text { months)/ } \\
2+1\end{array}$ & $\begin{array}{l}2004-2005 \\
2007\end{array}$ & $\begin{array}{l}<1 \text { year } \\
1 \text { year } \\
2-4 \text { years } \\
<5 \text { years } \\
\text { Overall }\end{array}$ & $\begin{array}{l}\text { All-type IPD; } \\
\text { VT IPD; } \\
\text { non-VT IPD }\end{array}$ \\
\hline $\begin{array}{l}\text { Vestrheim } 2010 \\
{[16], \text { Norway }}\end{array}$ & $\begin{array}{l}\text { National } \\
\text { Surveillance } \\
\text { System for } \\
\text { Communicable } \\
\text { Diseases }\end{array}$ & $\begin{array}{l}\text { PCV7 in NIP in } 2006 \\
\text { with catch-up (for } \\
\text { children } 3-6 \text { months)/ } \\
2+1\end{array}$ & $\begin{array}{l}2004-2005 \\
2008\end{array}$ & $\begin{array}{l}<5 \text { years } \\
5-19 \text { years } \\
20-39 \text { years } \\
40-64 \text { years } \\
\geq 65 \text { years } \\
\geq 5 \text { years } \\
\text { Overall }\end{array}$ & $\begin{array}{l}\text { All-type IPD; } \\
\text { VT IPD; } \\
\text { non-VT IPD }\end{array}$ \\
\hline
\end{tabular}


Table 1 continued

\begin{tabular}{|c|c|c|c|c|c|}
\hline $\begin{array}{l}\text { Study ID [ref], } \\
\text { country (specific } \\
\text { area) }\end{array}$ & Data sources & $\begin{array}{l}\text { PCV vaccination } \\
\text { policy/schedule }\end{array}$ & $\begin{array}{l}\text { Prevaccination/ } \\
\text { postvaccination time } \\
\text { periods }\end{array}$ & $\begin{array}{l}\text { Age groups } \\
\text { analyzed }\end{array}$ & $\begin{array}{l}\text { Outcomes } \\
\text { analyzed }\end{array}$ \\
\hline $\begin{array}{l}\text { Ardanuy } 2009 \\
{[17], \text { Spain }} \\
\text { (one hospital } \\
\text { in Southern } \\
\text { Barcelona) }\end{array}$ & $\begin{array}{l}\text { Single hospital, } \\
\text { prospective study } \\
\text { from } 1997 \text { to } \\
2007\end{array}$ & $\begin{array}{l}\text { PCV7 introduced } \\
\text { in } 2001 \text {, but in NIP } \\
\text { only for high-risk } \\
\text { children } / 3+1\end{array}$ & $\begin{array}{l}1997-2001 \\
2002-2004 \\
2005-2006\end{array}$ & $\begin{array}{l}18-64 \text { years } \\
\geq 65 \text { years } \\
\geq 18 \text { years }\end{array}$ & $\begin{array}{l}\text { All-type IPD; } \\
\text { VT IPD; } \\
\text { non-VT IPD }\end{array}$ \\
\hline $\begin{array}{l}\text { Aristegui } 2007 \\
{[18], \text { Spain }} \\
\text { (Basque Country } \\
\text { and Navarre) }\end{array}$ & $\begin{array}{l}\text { Retrospective } \\
\text { and prospective } \\
\text { data from nine } \\
\text { hospitals }\end{array}$ & $\begin{array}{l}\text { PCV7 introduced in } \\
2001, \text { but only private } \\
\text { market } 3+1\end{array}$ & $\begin{array}{l}1998-2001 \\
2002-2003\end{array}$ & $\begin{array}{l}<1 \text { year } \\
1-2 \text { years } \\
<2 \text { years } \\
2-5 \text { years } \\
<5 \text { years }\end{array}$ & $\begin{array}{l}\text { All-type IPD; } \\
\text { VT IPD; } \\
\text { non-VT IPD }\end{array}$ \\
\hline $\begin{array}{l}\text { Barricate } 2007 \\
\text { [19], Spain }\end{array}$ & $\begin{array}{l}\text { Data from all } \\
\text { laboratories in the } \\
\text { region }\end{array}$ & $\begin{array}{l}\text { PCV7 introduced in } \\
2001 \text {, but only private } \\
\text { market } / 3+1\end{array}$ & $\begin{array}{l}2000-2002 \\
2004-2005\end{array}$ & $\begin{array}{l}<2 \text { years } \\
<5 \text { years }\end{array}$ & All-type IPD \\
\hline $\begin{array}{l}\text { Calbo } 2006 \\
\text { [20], Spain (two } \\
\text { hospitals in one } \\
\text { health district in } \\
\text { Barcelona region) }\end{array}$ & $\begin{array}{l}\text { Data from two } \\
\text { hospitals }\end{array}$ & $\begin{array}{l}\text { PCV7 introduced in } \\
2001, \text { but only private } \\
\text { market } 3+1\end{array}$ & $\begin{array}{l}1999-2001 \\
2002-2004\end{array}$ & $\leq 5$ years & All-type IPD \\
\hline $\begin{array}{l}\text { Fenoll } 2009[21] \text {, } \\
\text { Spain }\end{array}$ & $\begin{array}{l}\text { Data from } \\
\text { National } \\
\text { Reference } \\
\text { Laboratory (from } \\
190 \text { hospitals in } \\
\text { country) }\end{array}$ & $\begin{array}{l}\text { PCV7 introduced in } \\
2001, \text { but only private } \\
\text { market } 3+1\end{array}$ & $\begin{array}{l}1996-2001 \\
2005-2006\end{array}$ & $\begin{array}{l}\text { Global (no } \\
\text { age groups) }\end{array}$ & All-type IPD \\
\hline $\begin{array}{l}\text { Guevara } 2009[22] \text {, } \\
\text { Spain (Navarre) }\end{array}$ & $\begin{array}{l}\text { Active surveillance } \\
\text { with participation } \\
\text { of all six } \\
\text { laboratories in the } \\
\text { region }\end{array}$ & $\begin{array}{l}\text { PCV7 introduced in } \\
2001, \text { but only private } \\
\text { market } / 3+1\end{array}$ & $\begin{array}{l}2001-2002 \\
2003-2005 \\
2006-2007\end{array}$ & $\begin{array}{l}\text { Global (no } \\
\text { age groups) }\end{array}$ & $\begin{array}{l}\text { All-type IPD; } \\
\text { VT IPD; } \\
\text { non-VT IPD }\end{array}$ \\
\hline $\begin{array}{l}\text { Munoz-Almagro } \\
2008 \text { [23], Spain } \\
\text { (one hospital } \\
\text { in Southern } \\
\text { Barcelona) }\end{array}$ & $\begin{array}{l}\text { Prospective study } \\
\text { in one hospital }\end{array}$ & $\begin{array}{l}\text { PCV7 introduced in } \\
2001 \text {, but only private } \\
\text { market } 3+1\end{array}$ & $\begin{array}{l}1997-2001 \\
2002-2006\end{array}$ & $\begin{array}{l}<2 \text { years } \\
2-4 \text { years } \\
5-17 \text { years }\end{array}$ & $\begin{array}{l}\text { All-type IPD; } \\
\text { VT IPD; } \\
\text { non-VT IPD }\end{array}$ \\
\hline $\begin{array}{l}\text { Perez-Trallero } \\
2009 \text { [24], Spain } \\
\text { (Gipuzkoa } \\
\text { province, Basque } \\
\text { Country) }\end{array}$ & $\begin{array}{l}\text { One hospital } \\
\text { (referral hospital } \\
\text { for whole region) }\end{array}$ & $\begin{array}{l}\text { PCV7 introduced in } \\
2001, \text { but only private } \\
\text { market } / 3+1\end{array}$ & $\begin{array}{l}1996-2001 \text { (pre for } \\
<5 \text { years) } \\
1998-2001 \text { (pre for } \\
>64 \text { years) } \\
2002-2007 \text { (for both) }\end{array}$ & $\begin{array}{l}<5 \text { years } \\
>64 \text { years }\end{array}$ & All-type IPD \\
\hline
\end{tabular}


Table 1 continued

\begin{tabular}{|c|c|c|c|c|c|}
\hline $\begin{array}{l}\text { Study ID [ref], } \\
\text { country (specific } \\
\text { area) }\end{array}$ & Data sources & $\begin{array}{l}\text { PCV vaccination } \\
\text { policy/schedule }\end{array}$ & $\begin{array}{l}\text { Prevaccination/ } \\
\text { postvaccination time } \\
\text { periods }\end{array}$ & $\begin{array}{l}\text { Age groups } \\
\text { analyzed }\end{array}$ & $\begin{array}{l}\text { Outcomes } \\
\text { analyzed }\end{array}$ \\
\hline $\begin{array}{l}\text { Foster } 2011 \text { [25], } \\
\text { UK (Oxfordshire) }\end{array}$ & $\begin{array}{l}\text { Ongoing } \\
\text { surveillance } \\
\text { system }\end{array}$ & $\begin{array}{l}\text { PCV7 introduced in } \\
2006 / 2+1\end{array}$ & $\begin{array}{l}1995-2006 \text { and } 2003- \\
2006 / 2006-2009\end{array}$ & $\begin{array}{l}<2 \text { years } \\
\geq 2 \text { years } \\
\text { All age }\end{array}$ & $\begin{array}{l}\text { All-type IPD; } \\
\text { VT IPD; VRT } \\
\text { IPD; non-VT } \\
\text { IPD }\end{array}$ \\
\hline $\begin{array}{l}\text { Miller } 2011[26] \text {, } \\
\text { UK (England and } \\
\text { Wales) }\end{array}$ & $\begin{array}{l}\text { Laboratory reports } \\
\text { given to the HPA } \\
\text { and isolates sent to } \\
\text { HPA reference lab } \\
\text { for serotyping }\end{array}$ & $\begin{array}{l}\text { PCV7 introduced in } \\
2006 / 2+1\end{array}$ & $\begin{array}{l}2000-2006 / \\
2009-2010\end{array}$ & $\begin{array}{l}<2 \text { years } \\
2-4 \text { years } \\
5-14 \text { years } \\
15-44 \text { years } \\
45-64 \text { years } \\
\geq 65 \text { years } \\
\text { All ages }\end{array}$ & $\begin{array}{l}\text { All-type IPD; } \\
\text { VT IPD; } \\
\text { non-VT IPD }\end{array}$ \\
\hline $\begin{array}{l}\text { Albrich } 2007 \text { [27], } \\
\text { USA (Georgia) }\end{array}$ & $\begin{array}{l}\text { Active laboratory } \\
\text { and population- } \\
\text { based surveillance } \\
\text { systems from } \\
\text { CDC-sponsored } \\
\text { Georgia Emerging } \\
\text { Infections Program }\end{array}$ & $\begin{array}{l}\text { PCV7 introduced in } \\
2000 / 3+1\end{array}$ & $\begin{array}{l}\text { 1997-2000 } \\
\text { (June)/2000 (July)- } \\
2004\end{array}$ & $\begin{array}{l}\leq 4 \text { years } \\
5-17 \text { years } \\
18-39 \text { years } \\
40-64 \text { years } \\
\geq 65 \text { years } \\
<18 \text { years } \\
\geq 18 \text { years }\end{array}$ & All-type IPD \\
\hline $\begin{array}{l}\text { Black } 2007 \text { [28], } \\
\text { USA (NCKP) }\end{array}$ & NCKP database & $\begin{array}{l}\text { PCV7 introduced in } \\
2000 / 3+1\end{array}$ & $\begin{array}{l}\text { 1996-2000 } \\
(\text { March)/2000 } \\
\text { (April)-2005 }\end{array}$ & $\begin{array}{l}<5 \text { years } \\
<6 \text { months } \\
6-23 \\
\text { months } \\
2-5 \text { years }\end{array}$ & $\begin{array}{l}\text { All-type IPD; } \\
\text { VT IPD; } \\
\text { non-VT IPD }\end{array}$ \\
\hline $\begin{array}{l}\text { Whitney } 2003 \\
\text { [29], USA (ABCs } \\
\text { in eight states) }\end{array}$ & $\begin{array}{l}\text { Active surveillance } \\
\text { system }(\mathrm{ABCs})^{\mathrm{a}}\end{array}$ & $\begin{array}{l}\text { PCV7 introduced in } \\
2000 / 3+1\end{array}$ & $1998-1999 / 2001$ & $\begin{array}{l}<1 \text { year } \\
1 \text { year } \\
2 \text { years } \\
<2 \text { years } \\
5-19 \text { years } \\
20-39 \text { years } \\
40-64 \text { years } \\
\geq 65 \text { years }\end{array}$ & $\begin{array}{l}\text { All-type IPD; } \\
\text { VT IPD; } \\
\text { non-VT IPD; } \\
\text { VRT IPD }\end{array}$ \\
\hline $\begin{array}{l}\text { Lexau } 2005[30] \text {, } \\
\text { USA (ABCs in } \\
\text { eight states) }\end{array}$ & $\begin{array}{l}\text { Active surveillance } \\
\text { system }(\mathrm{ABCs})^{\mathrm{a}}\end{array}$ & $\begin{array}{l}\text { PCV7 introduced in } \\
2000 / 3+1\end{array}$ & $\begin{array}{l}1998-1999 / \\
2000-2001 / \\
2002-2003\end{array}$ & $\begin{array}{l}50-64 \text { years } \\
65-74 \text { years } \\
75-84 \text { years } \\
\geq 85 \text { years }\end{array}$ & $\begin{array}{l}\text { All-type IPD; } \\
\text { VT IPD; } \\
\text { non-VT IPD; } \\
\text { STs only in } \\
\text { polysaccharide } \\
\text { vaccine }\end{array}$ \\
\hline $\begin{array}{l}\text { Hicks } 2007 \text { [31], } \\
\text { USA (ABCs in } \\
\text { eight states) }\end{array}$ & $\begin{array}{l}\text { Active surveillance } \\
\text { system }(\mathrm{ABCs})^{\mathrm{a}}\end{array}$ & $\begin{array}{l}\text { PCV7 introduced in } \\
2000 / 3+1\end{array}$ & $1998-1999 / 2004$ & $\begin{array}{l}<5 \text { years } \\
\geq 65 \text { years }\end{array}$ & $\begin{array}{l}\text { All-type IPD; } \\
\text { VT IPD; } \\
\text { non-VT IPD }\end{array}$ \\
\hline
\end{tabular}


Table 1 continued

\begin{tabular}{|c|c|c|c|c|c|}
\hline $\begin{array}{l}\text { Study ID [ref], } \\
\text { country (specific } \\
\text { area) }\end{array}$ & Data sources & $\begin{array}{l}\text { PCV vaccination } \\
\text { policy/schedule }\end{array}$ & $\begin{array}{l}\text { Prevaccination/ } \\
\text { postvaccination time } \\
\text { periods }\end{array}$ & $\begin{array}{l}\text { Age groups } \\
\text { analyzed }\end{array}$ & $\begin{array}{l}\text { Outcomes } \\
\text { analyzed }\end{array}$ \\
\hline $\begin{array}{l}\text { CDC } 2008 \text { [32], } \\
\text { USA (ABCs in } \\
\text { eight states) }\end{array}$ & $\begin{array}{l}\text { Active surveillance } \\
\text { system }(\mathrm{ABCs})^{\mathrm{a}}\end{array}$ & $\begin{array}{l}\text { PCV7 introduced in } \\
2000 / 3+1\end{array}$ & $1998-1999 / 2005$ & $\begin{array}{l}<1 \text { year } \\
1 \text { year } \\
2 \text { years } \\
3 \text { years } \\
4 \text { years } \\
<5 \text { years }\end{array}$ & $\begin{array}{l}\text { All-type IPD; } \\
\text { VT IPD; } \\
\text { non-VT IPD }\end{array}$ \\
\hline $\begin{array}{l}\text { Pilishvili } 2010 \\
\text { [33], USA (ABCs } \\
\text { in eight states) }\end{array}$ & $\begin{array}{l}\text { Active surveillance } \\
\text { system }(\mathrm{ABCs})^{\mathrm{a}}\end{array}$ & $\begin{array}{l}\text { PCV7 introduced in } \\
2000 / 3+1\end{array}$ & $1998-1999 / 2007$ & $\begin{array}{l}<5 \text { years } \\
5-17 \text { years } \\
18-49 \text { years } \\
50-64 \text { years } \\
\geq 65 \text { years }\end{array}$ & $\begin{array}{l}\text { All-type IPD; } \\
\text { VT IPD; } \\
\text { non-VT IPD; } \\
\text { 19A IPD; } \\
\text { VRT IPD }\end{array}$ \\
\hline $\begin{array}{l}\text { Hennessy } 2005 \\
\text { [34], USA (Alaska) }\end{array}$ & $\begin{array}{l}\text { Active surveillance } \\
\text { system in Alaska, } \\
\text { run by CDC }\end{array}$ & $\begin{array}{l}\text { PCV7 introduced in } \\
2000 / 3+1\end{array}$ & $\begin{array}{l}1995-2000 / \\
2001-2003\end{array}$ & $\begin{array}{l}<2 \text { years } \\
2-4 \text { years } \\
5-17 \text { years } \\
18-44 \text { years } \\
\geq 45 \text { years }\end{array}$ & $\begin{array}{l}\text { All-type IPD; } \\
\text { VT IPD; } \\
\text { non-VT IPD }\end{array}$ \\
\hline $\begin{array}{l}\text { Singleton } 2007 \\
\text { [35], USA (Alaska) }\end{array}$ & $\begin{array}{l}\text { Active surveillance } \\
\text { system in Alaska, } \\
\text { run by CDC }\end{array}$ & $\begin{array}{l}\text { PCV7 introduced in } \\
2000 / 3+1\end{array}$ & $\begin{array}{l}1995-2000 / \\
2001-2003 / \\
2004-2006\end{array}$ & $\begin{array}{l}<2 \text { years } \\
2-4 \text { years } \\
5-17 \text { years } \\
18-44 \text { years } \\
\geq 45 \text { years } \\
\text { Global }\end{array}$ & $\begin{array}{l}\text { All-type IPD; } \\
\text { VT IPD; } \\
\text { non-VT IPD }\end{array}$ \\
\hline $\begin{array}{l}\text { Hsu } 2005 \\
\text { [36], USA } \\
\text { (Massachusetts) }\end{array}$ & $\begin{array}{l}\text { Passive laboratory } \\
\text { surveillance with } \\
\text { calls to primary care } \\
\text { provider or parents }\end{array}$ & $\begin{array}{l}\text { PCV7 introduced in } \\
2000 / 3+1\end{array}$ & $\begin{array}{l}1990-1991 / \\
2001-2003\end{array}$ & $<5$ years & $\begin{array}{l}\text { All-type IPD; } \\
\text { VT IPD; } \\
\text { non-VT IPD }\end{array}$ \\
\hline $\begin{array}{l}\text { McBean } 2005 \text { [37], } \\
\text { USA (Medicare) }\end{array}$ & $\begin{array}{l}\text { Medicare analysis } \\
\text { and review files }\end{array}$ & $\begin{array}{l}\text { PCV7 introduced in } \\
2002 / 3+1\end{array}$ & $\begin{array}{l}1996-2000 / \\
2000-2001 / \\
2001-2002 / \\
2002-2003\end{array}$ & $\begin{array}{l}65-74 \text { years } \\
75-84 \text { years } \\
\geq 85 \text { years }\end{array}$ & $\begin{array}{l}\text { All-type IPD } \\
\text { (ICD-9-CM } \\
\text { codes) }\end{array}$ \\
\hline $\begin{array}{l}\text { Pulido } 2010 \text { [38], } \\
\text { USA (national) }\end{array}$ & $\begin{array}{l}\text { National Center } \\
\text { for Health } \\
\text { Statistics data files } \\
\text { on cause of death } \\
\text { (ICD-9 and ICD- } \\
10 \text { codes) }\end{array}$ & $\begin{array}{l}\text { PCV7 introduced in } \\
2002 / 3+1\end{array}$ & $\begin{array}{l}1990-1998 / \\
2001-2005\end{array}$ & $\begin{array}{l}<2 \text { years } \\
2-4 \text { years } \\
5-14 \text { years } \\
15-24 \text { years } \\
25-34 \text { years } \\
35-44 \text { years } \\
45-54 \text { years } \\
55-64 \text { years } \\
65-74 \text { years } \\
75-84 \text { years } \\
\geq 85 \text { years }\end{array}$ & $\begin{array}{l}\text { IPD mortality } \\
\text { (all type) }\end{array}$ \\
\hline
\end{tabular}


Table 1 continued

\begin{tabular}{|c|c|c|c|c|c|}
\hline $\begin{array}{l}\text { Study ID [ref], } \\
\text { country (specific } \\
\text { area) }\end{array}$ & Data sources & $\begin{array}{l}\text { PCV vaccination } \\
\text { policy/schedule }\end{array}$ & $\begin{array}{l}\text { Prevaccination/ } \\
\text { postvaccination time } \\
\text { periods }\end{array}$ & $\begin{array}{l}\text { Age groups } \\
\text { analyzed }\end{array}$ & $\begin{array}{l}\text { Outcomes } \\
\text { analyzed }\end{array}$ \\
\hline $\begin{array}{l}\text { Simonsen } 2011 \\
\text { [39], USA } \\
\text { (10 states) }\end{array}$ & $\begin{array}{l}\text { Health Care } \\
\text { Utilization Project } \\
\text { State Inpatient } \\
\text { Databases with } \\
\text { ICD-9 codes }\end{array}$ & $\begin{array}{l}\text { PCV7 introduced in } \\
2002 / 3+1\end{array}$ & $\begin{array}{l}1996-1997 / \\
2005-2006\end{array}$ & $\begin{array}{l}<2 \text { years } \\
2-4 \text { years } \\
5-17 \text { years } \\
18-39 \text { years } \\
40-64 \text { years } \\
\geq 65 \text { years }\end{array}$ & $\begin{array}{l}\text { All-type } \\
\text { IPD; all- } \\
\text { type IPD } \\
\text { mortality }\end{array}$ \\
\hline $\begin{array}{l}\text { Tsigrelis } 2008 \text { [40], } \\
\text { USA (Minnesota) }\end{array}$ & $\begin{array}{l}\text { Two laboratory } \\
\text { databases }\end{array}$ & $\begin{array}{l}\text { PCV7 introduced in } \\
2002 / 3+1\end{array}$ & $\begin{array}{l}1995-1999 / \\
2001-2007\end{array}$ & $\begin{array}{l}<5 \text { years } \\
5-19 \text { years } \\
20-39 \text { years } \\
40-64 \text { years } \\
\geq 65 \text { years }\end{array}$ & $\begin{array}{l}\text { All-type } \\
\text { IPD } \\
\text { mortality }\end{array}$ \\
\hline $\begin{array}{l}\text { Tyrrell } 2009 \text { [41], } \\
\text { Canada (Alberta) }\end{array}$ & $\begin{array}{l}\text { National Center } \\
\text { for Streptococcus } \\
\text { database }\end{array}$ & $\begin{array}{l}\text { PCV7 introduced in } \\
2002 / 2+1\end{array}$ & $2000 / 2006$ & $\begin{array}{l}<2 \text { years } \\
2-4 \text { years } \\
5-19 \text { years } \\
20-39 \text { years } \\
40-64 \text { years } \\
\geq 65 \text { years }\end{array}$ & $\begin{array}{l}\text { All-type } \\
\text { IPD; VT } \\
\text { IPD; } \\
\text { non-VT } \\
\text { IPD }\end{array}$ \\
\hline $\begin{array}{l}\text { Kellner } 2009 \text { [42], } \\
\text { Canada (Calgary) }\end{array}$ & $\begin{array}{l}\text { Prospective, } \\
\text { population-based } \\
\text { surveillance } \\
\text { system }\end{array}$ & $\begin{array}{l}\text { PCV7 introduced in } \\
2002 / 2+1\end{array}$ & $\begin{array}{l}1998-2001 / \\
2003-2007\end{array}$ & $\begin{array}{l}0-6 \text { months } \\
6-23 \\
\text { months } \\
2-4 \text { years } \\
5-15 \text { years } \\
16-64 \text { years } \\
65-84 \text { years } \\
\geq 85 \text { years }\end{array}$ & $\begin{array}{l}\text { All-type } \\
\text { IPD; VT } \\
\text { IPD; } \\
\text { non-VT } \\
\text { IPD }\end{array}$ \\
\hline $\begin{array}{l}\text { Hanna } 2010 \text { [43], } \\
\text { Australia (North } \\
\text { Queensland) }\end{array}$ & $\begin{array}{l}\text { Prospective } \\
\text { surveillance } \\
\text { system (obligatory } \\
\text { notification) }\end{array}$ & $\begin{array}{l}\text { PCV7 (and PPV23) } \\
\text { introduced universally } \\
\text { in } 2005 / 2+1\end{array}$ & $\begin{array}{l}2001-2004 / \\
2006-2009\end{array}$ & $\begin{array}{l}<5 \text { years } \\
5-14 \text { years } \\
15-64 \text { years } \\
\text { All ages }\end{array}$ & $\begin{array}{l}\text { All-type } \\
\text { IPD; VT } \\
\text { IPD; for } \\
\geq 15 \text { years: } \\
\text { ST common } \\
\text { to both } \\
\text { and only in } \\
\text { PPV23 }\end{array}$ \\
\hline $\begin{array}{l}\text { Lehmann } 2010 \\
\text { [44], Australia } \\
\text { (Western } \\
\text { Australia) }\end{array}$ & $\begin{array}{l}\text { Prospective } \\
\text { surveillance } \\
\text { system (obligatory } \\
\text { notification) }\end{array}$ & $\begin{array}{l}\text { PCV7 (and PPV23) } \\
\text { introduced universally } \\
\text { in } 2005 / 2+1 \text { (in } 1999 \\
\text { and } 2001 \text {, respectively, } \\
\text { for aboriginals) }\end{array}$ & $\begin{array}{l}1997-2001 / \\
2002-2004 / \\
2005-2007\end{array}$ & $\begin{array}{l}<2 \text { years } \\
2-4 \text { years } \\
5-14 \text { years } \\
15-29 \text { years } \\
30-49 \text { years } \\
50-64 \text { years } \\
\geq 65 \text { years }\end{array}$ & $\begin{array}{l}\text { All-type } \\
\text { IPD; VT } \\
\text { IPD; } \\
\text { non-VT } \\
\text { IPD }\end{array}$ \\
\hline
\end{tabular}


Table 1 continued

\begin{tabular}{|c|c|c|c|c|c|}
\hline $\begin{array}{l}\text { Study ID [ref], } \\
\text { country (specific } \\
\text { area) }\end{array}$ & Data sources & $\begin{array}{l}\text { PCV vaccination } \\
\text { policy/schedule }\end{array}$ & $\begin{array}{l}\text { Prevaccination/ } \\
\text { postvaccination time } \\
\text { periods }\end{array}$ & $\begin{array}{l}\text { Age groups } \\
\text { analyzed }\end{array}$ & $\begin{array}{l}\text { Outcomes } \\
\text { analyzed }\end{array}$ \\
\hline $\begin{array}{l}\text { Roche } 2008 \\
\text { [45], Australia } \\
\text { (national) }\end{array}$ & $\begin{array}{l}\text { Prospective } \\
\text { surveillance } \\
\text { system (obligatory } \\
\text { notification) } \\
\text { in National } \\
\text { Notifiable Diseases } \\
\text { Surveillance } \\
\text { System }\end{array}$ & $\begin{array}{l}\text { PCV7 (and PPV23) } \\
\text { introduced universally } \\
\text { in } 2005 / 2+1 \text { (in } 1999 \\
\text { and } 2001 \text {, respectively, } \\
\text { for aboriginals) }\end{array}$ & - & $\begin{array}{l}<2 \text { years } \\
2-14 \text { years } \\
15-49 \text { years } \\
50-64 \text { years } \\
\geq 65 \text { years }\end{array}$ & $\begin{array}{l}\text { All-type } \\
\text { IPD; VT } \\
\text { IPD; } \\
\text { non-VT } \\
\text { IPD; IPD } \\
\text { mortality }\end{array}$ \\
\hline $\begin{array}{l}\text { Williams } 2011 \\
\text { [46], Australia } \\
\text { (national) }\end{array}$ & $\begin{array}{l}\text { Prospective } \\
\text { surveillance } \\
\text { system (obligatory } \\
\text { notification) } \\
\text { in National } \\
\text { Notifiable Diseases } \\
\text { Surveillance } \\
\text { System }\end{array}$ & $\begin{array}{l}\text { PCV7 (and PPV23) } \\
\text { introduced universally } \\
\text { in } 2005 / 2+1 \text { (in } 1999 \\
\text { and } 2001 \text {, respectively, } \\
\text { for aboriginals) }\end{array}$ & $\begin{array}{l}2002-2004 / \\
2006 / 2007\end{array}$ & $<2$ years & $\begin{array}{l}\text { All-type } \\
\text { IPD; VT } \\
\text { IPD; } \\
\text { non-VT } \\
\text { IPD }\end{array}$ \\
\hline
\end{tabular}

$A B C$ s active bacterial core surveillance, $C D C$ Centers for Disease Control and Prevention, $H P A$ Health Protection Agency, ICD-9-CM International Statistical Classification of Diseases and Health-related Problems, 9th Revision clinically modified, ICD-10-CM International Statistical Classification of Diseases and Health-related Problems, 10th Revision clinically modified, IPD invasive pneumococcal disease, NCKP North Carolina Kaiser Permanente, $N I P$ national immunization program, non-VT IPD nonvaccine-type IPD, $P C V$ pneumococcal conjugate vaccine, $P P V 23$ 23-valent pneumococcal polysaccharide vaccine, $S T$ subtype, $V T$ IPD vaccine-type IPD, VRT IPD vaccinerelated-type IPD

${ }^{a} \mathrm{ABC}$ s is run by the CDC and covers a population of 29,757,552 persons in California (San Francisco County and children < 5 years in Alameda and Contra Costa counties); Colorado (five county Denver area); Connecticut; Georgia (20 county Atlanta area); Maryland (six county Baltimore area); Minnesota; New Mexico; New York (15 county Rochester and Albany areas and children $<5$ years in Erie county); Oregon (three county Portland area); Tennessee (20 counties)

but analyzed different post-PCV vaccination periods [34, 35]. Two of the North American studies were from Canada $[41,42]$. Four studies were from Australia [43-46].

\section{Impact on VT IPD in Vaccine-Eligible Children}

The impact of PCV7 on VT IPD in vaccine-eligible children ( $<2$ or $<5$ years old, depending on the study) was evaluated in 18 studies (Table 2). A reduction in VT IPD incidence was reported in all the studies, ranging from $39.9 \%$ in
Spain [23] to $99.1 \%$ in the USA [32]; the median rate reduction was $90.1 \%$.

\section{Vaccine Impact on All-Type IPD in Vaccine- Eligible Children}

The impact of PCV7 on all-type IPD in vaccine-eligible children $(<2$ years or $<5$ years depending on the study) was evaluated in 30 studies (three from Australia, 15 from Europe, and 12 from North America) (Table 3). All but one of the studies reported a reduction 
Table 2 Impact on VT IPD in vaccine-eligible populations

\begin{tabular}{|c|c|c|c|c|c|c|}
\hline \multirow{2}{*}{$\begin{array}{l}\text { Study (year of PCV7 } \\
\text { implementation) [ref] }\end{array}$} & \multirow{2}{*}{$\begin{array}{l}\text { Study } \\
\text { population }\end{array}$} & \multicolumn{2}{|c|}{ Prevaccination } & \multicolumn{2}{|c|}{ Postvaccination } & \multirow[t]{2}{*}{$\%$ change } \\
\hline & & Years & $\begin{array}{l}\text { Incidence/ } \\
100,000\end{array}$ & Years & $\begin{array}{l}\text { Incidence/ } \\
100,000\end{array}$ & \\
\hline \multicolumn{7}{|c|}{ Australia (January 2005 for all <2 years) } \\
\hline Lehmann 2010 [44] & $\begin{array}{l}\text { Nonaboriginal, } \\
<2 \text { years (PCV7 } \\
\text { and PPV23) }\end{array}$ & $1997-2001$ & 61.2 & $2005-2007$ & 6.6 & -89.2 \\
\hline Roche 2008 [45] & $\begin{array}{l}\text { Nonindigenous } \\
<2 \text { years }\end{array}$ & 2004 & 73.4 & 2006 & 6.7 & -90.9 \\
\hline Williams 2011 [46] & $<2$ years & $2002-2004$ & 60.9 & 2007 & 2.1 & -96.6 \\
\hline \multicolumn{7}{|l|}{ Europe } \\
\hline $\begin{array}{l}\text { Foster 2010, UK (September } \\
2006)[25]\end{array}$ & $<2$ years & $2003-2005$ & 26.4 & $2006-2009$ & 5.5 & -79.2 \\
\hline $\begin{array}{l}\text { Miller 2011, UK (September } \\
\text { 2006) [26] }\end{array}$ & $<2$ years & $2000-2006$ & 40.8 & $2009-2010$ & 0.9 & -97.8 \\
\hline Vestrheim 2008, Norway & $<1$ year & $2004-2005$ & 40.5 & 2007 & 3.4 & -91.6 \\
\hline$(2006)[15]$ & 1 year & & 53.7 & & 24.3 & -54.7 \\
\hline $\begin{array}{l}\text { Hanquet 2011, Belgium } \\
\text { (January 2007) [11] }\end{array}$ & $<2$ years & $2002-2003$ & 92.9 & 2008 & 4.0 & -95.7 \\
\hline $\begin{array}{l}\text { Harboe 2010, Denmark } \\
(2007)[12]\end{array}$ & $<2$ years & $2000-2007$ & 36.7 & 2008 & 7.7 & -79.0 \\
\hline $\begin{array}{l}\text { Munoz-Almagro 2008, Spain } \\
\text { (2001) [23] }\end{array}$ & $<2$ years & $1997-2001$ & 26.8 & $2002-2006$ & 16.1 & -39.9 \\
\hline $\begin{array}{l}\text { Barricarte 2007, Spain } \\
(2001)[19]\end{array}$ & $<2$ years & $2000-2002$ & 56.0 & $2004-2005$ & 16.0 & -71.4 \\
\hline $\begin{array}{l}\text { Rodenburg 2010, Netherlands } \\
\text { (June 2006) [14] }\end{array}$ & $<2$ years & $2004-2006$ & 24.3 & $2006-2008$ & 8.0 & -67.1 \\
\hline \multicolumn{7}{|c|}{ North America (2001 in USA and 2002 in Alberta, Canada) } \\
\hline Hennessy 2005 [34] & $\begin{array}{l}\text { Nonnative } \\
<2 \text { years }\end{array}$ & $1995-2000$ & 101.3 & $2001-2003$ & 20.0 & -80.3 \\
\hline Singleton 2007 [35] & $\begin{array}{l}\text { Nonnative } \\
<2 \text { years }\end{array}$ & $1995-2000$ & 101.3 & $2004-2006$ & 2.3 & -97.7 \\
\hline Whitney 2003 [29] & $<2$ years & 1998-1999 & 156.1 & 2001 & 33.6 & -78.5 \\
\hline \multirow[t]{2}{*}{ CDC 2008 [32] } & 1 year & 1998-1999 & 177.3 & 2005 & 1.6 & -99.1 \\
\hline & $<1$ year & & 144.0 & & 2.7 & -98.1 \\
\hline Pilishvili 2010 [33] & $<5$ years & 1998-1999 & 81.9 & 2007 & 0.4 & -99.5 \\
\hline Tyrrell 2009 [41] & $<2$ years & 2000 & 86.3 & 2006 & 4.7 & -94.6 \\
\hline Kellner 2009 [42] & $<2$ years & $1998-2001$ & 66.4 & 2003-2007 & 9.0 & -86.4 \\
\hline
\end{tabular}

$C D C$ Centers for Disease Control and Prevention, $P C V 77$-valent pneumococcal conjugate vaccine, PPV23 23-valent pneumococcal polysaccharides vaccine, $V T$ IPD vaccine-type invasive pneumococcal disease 
Table 3 Impact on all-type IPD reduction in vaccine eligible population (continued on next page)

\begin{tabular}{|c|c|c|c|c|c|c|}
\hline \multirow{2}{*}{$\begin{array}{l}\text { Study (year of PCV7 } \\
\text { implementation) [ref] }\end{array}$} & \multirow{2}{*}{$\begin{array}{l}\text { Study } \\
\text { population }\end{array}$} & \multicolumn{2}{|c|}{ Prevaccination } & \multicolumn{2}{|c|}{ Postvaccination } & \multirow[t]{2}{*}{$\%$ change } \\
\hline & & Years & $\begin{array}{l}\text { Incidence/ } \\
100,000\end{array}$ & Years & $\begin{array}{l}\text { Incidence/ } \\
100,000\end{array}$ & \\
\hline \multicolumn{7}{|l|}{ Australia (January 2005 for all) } \\
\hline Lehmann $2010[44]$ & $\begin{array}{l}\text { Nonaboriginal } \\
<2 \text { years }\end{array}$ & $1997-2001$ & 73.8 & $2005-2007$ & 24.2 & -67.2 \\
\hline Roche 2006 [45] & $\begin{array}{l}\text { General } \\
\text { population } \\
<2 \text { years }\end{array}$ & 2002 & 93.0 & 2006 & 22.0 & -76.3 \\
\hline Williams 2011 [46] & $<2$ years & 2002 & 98.1 & 2007 & 25.1 & -74.4 \\
\hline \multicolumn{7}{|l|}{ Europe } \\
\hline $\begin{array}{l}\text { Verstrheim 2008, Norway } \\
\text { (2006) [15] }\end{array}$ & $\begin{array}{l}<2 \text { years } \\
<5 \text { years }\end{array}$ & $2004-2005$ & $\begin{array}{l}67.7 \\
36.0\end{array}$ & 2007 & $\begin{array}{l}32.6 \\
19.7\end{array}$ & $\begin{array}{l}-51.8 \\
-45.3\end{array}$ \\
\hline $\begin{array}{l}\text { Vestrheim 2010, Norway } \\
(2006)[16]\end{array}$ & $<5$ years & $2004-2005$ & 35.9 & 2008 & 9.9 & -72.4 \\
\hline $\begin{array}{l}\text { Harboe 2010, Denmark } \\
(2007)[12]\end{array}$ & $<2$ years & $2000-2007$ & 54.8 & 2008 & 23.8 & -56.6 \\
\hline Foster 2010, UK (September & $<2$ years & $1995-2005$ & 43.3 & $2006-2009$ & 22.4 & -48.3 \\
\hline 2006) $[25]$ & & $2003-2005$ & 35.9 & $2006-2009$ & 22.4 & -37.6 \\
\hline $\begin{array}{l}\text { Miller 2011, UK } \\
\text { (September 2006) [26] }\end{array}$ & $<2$ years & $2000-2006$ & 54.2 & $2009-2010$ & 23.6 & -56.5 \\
\hline $\begin{array}{l}\text { Rückinger } 2009, \text { Germany } \\
\text { (July 2006) [13] }\end{array}$ & $\begin{array}{l}<2 \text { years } \\
\text { (capture- } \\
\text { recapture) }\end{array}$ & $1997-2003$ & 20.0 & $2007-2008$ & 11.0 & -45.0 \\
\hline $\begin{array}{l}\text { Hanquet 2011, Belgium } \\
\text { (January 2007) [11] }\end{array}$ & $<2$ years & $2002-2003$ & 129.7 & 2008 & 82.4 & -36.5 \\
\hline $\begin{array}{l}\text { Rodenburg 2010, } \\
\text { Netherlands (June 2006) } \\
{[14]}\end{array}$ & $<2$ years & $2004-2006$ & 34.5 & 2006-2008 & 22.5 & -34.8 \\
\hline $\begin{array}{l}\text { Rendi-Wagner 2009, Austria } \\
\text { (2003) [10] }\end{array}$ & $<2$ years & 2001-2004 & 12.2 & $2004-2007$ & 8.7 & -28.7 \\
\hline $\begin{array}{l}\text { Aristegui } 2007, \text { Spain }(2001 \\
\text { - private) }[18]\end{array}$ & $<2$ years & $1998-2001$ & 93.5 & 2003 & 56.3 & -39.8 \\
\hline $\begin{array}{l}\text { Calbo 2006, Spain (2001) } \\
\text { [20] }\end{array}$ & $<5$ years & $1999-2001$ & 96.9 & $2002-2004$ & 90.6 & -6.5 \\
\hline $\begin{array}{l}\text { Munoz-Almagro } 2008 \\
\text { (2001 not national, or } \\
\text { reimbursed) [23] }\end{array}$ & $<2$ years & $1997-2001$ & 32.4 & $2002-2006$ & 51.3 & +58.3 \\
\hline $\begin{array}{l}\text { Perez-Trallero } 2009 \text { (late } \\
\text { 2001, not reimbursed) [24] }\end{array}$ & $<5$ years & $1996-2001$ & 50.0 & $2002-2007$ & 39.6 & -20.8 \\
\hline
\end{tabular}


Table 3 continued

\begin{tabular}{|c|c|c|c|c|c|c|}
\hline \multirow{2}{*}{$\begin{array}{l}\text { Study (year of PCV7 } \\
\text { implementation) [ref] }\end{array}$} & \multirow{2}{*}{$\begin{array}{l}\text { Study } \\
\text { population }\end{array}$} & \multicolumn{2}{|c|}{ Prevaccination } & \multicolumn{2}{|c|}{ Postvaccination } & \multirow[t]{2}{*}{$\%$ change } \\
\hline & & Years & $\begin{array}{l}\text { Incidence/ } \\
100,000\end{array}$ & Years & $\begin{array}{l}\text { Incidence/ } \\
100,000\end{array}$ & \\
\hline $\begin{array}{l}\text { Barricarte 2007, Spain } \\
(2001)[19]\end{array}$ & $<2$ years & $2000-2002$ & 117 & $2004-2005$ & 115 & -1.7 \\
\hline $\begin{array}{l}\text { Guevara 2009, Spain (June } \\
\text { 2001) [22] }\end{array}$ & $<5$ years & $2001-2002$ & 82.5 & $2006-2007$ & 72.4 & -12.2 \\
\hline \multicolumn{7}{|c|}{ North America (2001 in USA and 2002 in Canada) } \\
\hline Albrich 2007 [27] & $\leq 4$ years & $1997-2000$ & 139.7 & $2000-2004$ & 44.1 & -68.4 \\
\hline Black 2007 [28] & $<5$ years & $1996-2000$ & 62.5 & $2000-2005$ & 15.3 & -75.5 \\
\hline Whitney 2003 [29] & $<2$ years & $1998-1999$ & 188.0 & 2001 & 59.0 & -68.6 \\
\hline Hsu 2005 [36] & $<5$ years & $1990-1991$ & 56.9 & $2001-2003$ & 17.4 & -69.4 \\
\hline Hicks 2007 [31] & $<5$ years & $1998-1999$ & 95.2 & 2004 & 22.6 & -76.3 \\
\hline \multirow[t]{2}{*}{ CDC 2008 [32] } & 1 year & $1998-1999$ & 213.6 & 2005 & 37.8 & -82.3 \\
\hline & $<1$ year & & 170.5 & & 40.0 & -76.5 \\
\hline Pilishvili 2010 [33] & $<5$ years & $1998-1999$ & 98.7 & 2007 & 23.6 & -76.1 \\
\hline Kellner 2009 [42] & 6-23 months & $1998-2001$ & 77.7 & $2003-2007$ & 18.0 & -76.8 \\
\hline Tyrrell 2009 [41] & $<2$ years & 2000 & 96.7 & 2006 & 25.8 & -73.3 \\
\hline Hennessy 2005 [34] & $\begin{array}{l}\text { Nonnatives } \\
<2 \text { years }\end{array}$ & $1995-2000$ & 131.1 & $2001-2003$ & 51.0 & -61.1 \\
\hline Singleton 2007 [35] & $\begin{array}{l}\text { Nonnatives } \\
<2 \text { years }\end{array}$ & $1995-2000$ & 135.5 & $2004-2006$ & 43.6 & -67.8 \\
\hline \multirow[t]{2}{*}{ Simonsen 2011 [39] } & $<2$ years & $1996-1999$ & 27.8 & $2005-2006$ & 5.5 & -80.2 \\
\hline & $2-4$ years & & 5.4 & & 2.0 & -63.0 \\
\hline
\end{tabular}

$C D C$ Centers for Disease Control and Prevention, IPD invasive pneumococcal disease, $P C V 77$-valent pneumococcal conjugate vaccine

in all-type IPD incidence ranging from $1.7 \%$ (in Spain) [19] to $80.2 \%$ in the USA [32], with a median rate reduction of $45.0 \%$. The highest rate reductions were reported generally in North America, Australia, and Norway, and the lowest was in Spain; the only study that did not report a reduction was performed in a single hospital in Barcelona, Spain, which reported 198 episodes of IPD in 194 children, with an increase of 58.3\% between 1997-2001 and 2002-2006 [23].

\section{Impact on VT IPD in All Ages}

The impact of PCV7 on VT IPD in all ages was evaluated in nine studies that all reported a reduction ranging from $1.4 \%$ in the Netherlands [14] to $93.5 \%$ in the USA [33] with a median reduction rate of $65.5 \%$ (Table 4 ). In the study in the Netherlands, the periods compared were 20042006 and 2006-2008, so the postvaccination period was soon after the introduction of PCV7 into the NIP in 2006. 
Table 4 Impact on VT IPD reduction in all ages

\begin{tabular}{|c|c|c|c|c|c|c|}
\hline \multirow{2}{*}{$\begin{array}{l}\text { Study (year of PCV7 } \\
\text { implementation) [ref }]\end{array}$} & \multirow{2}{*}{$\begin{array}{l}\text { Study } \\
\text { population }\end{array}$} & \multicolumn{2}{|c|}{ Prevaccination } & \multicolumn{2}{|c|}{ Postvaccination } & \multirow[t]{2}{*}{$\%$ change } \\
\hline & & Years & $\begin{array}{l}\text { Incidence/ } \\
100,000\end{array}$ & Years & $\begin{array}{l}\text { Incidence/ } \\
100,000\end{array}$ & \\
\hline \multicolumn{7}{|c|}{ Australia (January 2005 for all) } \\
\hline Lehmann 2010 [44] & $\begin{array}{l}\text { Nonaboriginal, } \\
\text { all ages (PCV7 } \\
\& \text { PPV23) }\end{array}$ & $1997-2001$ & 5.3 & $2005-2007$ & 2.2 & -58.5 \\
\hline Hanna $2010[43]$ & $\begin{array}{l}\text { Nonindigenous } \\
\text { people, all ages }\end{array}$ & $2001-2004$ & 6.1 & 2006-2009 & 1.4 & -77.0 \\
\hline \multicolumn{7}{|l|}{ Europe } \\
\hline $\begin{array}{l}\text { Foster 2010, UK } \\
(\text { September 2006) [25] }\end{array}$ & All ages & $2003-2005$ & 4.3 & 2006-2009 & 2.2 & -48.8 \\
\hline $\begin{array}{l}\text { Miller } 2011 \\
\text { (September 2006) [26] }\end{array}$ & All ages & $2000-2006$ & 8.0 & $2009-2010$ & 1.1 & -86.3 \\
\hline $\begin{array}{l}\text { Fenoll 2009, Spain } \\
(\text { June 2001) [21] }\end{array}$ & $<15$ years & $1996-2001$ & 5.2 & $2005-2006$ & 2.4 & -53.8 \\
\hline $\begin{array}{l}\text { Guevara 2010, Spain } \\
\text { (June 2001) [22] }\end{array}$ & All ages & 2001-2002 & 5.5 & $2006-2007$ & 1.9 & -65.5 \\
\hline $\begin{array}{l}\text { Rodenburg 2010, } \\
\text { Netherlands } \\
\text { (June 2006) [14] }\end{array}$ & All ages & $2004-2006$ & 7.0 & $2006-2008$ & 6.9 & -1.4 \\
\hline \multicolumn{7}{|l|}{ North America (2001) } \\
\hline Singleton 2007 [35] & $\begin{array}{l}\text { All ages, } \\
\text { nonnatives }\end{array}$ & $1995-2000$ & 8.9 & 2004-2006 & 1.3 & -85.4 \\
\hline Pilishvili 2010 [33] & All ages & $1998-1999$ & 15.5 & 2007 & 1.0 & -93.5 \\
\hline
\end{tabular}

$I P D$ invasive pneumococcal disease, $P C V 77$-valent pneumococcal conjugate vaccine, $P P V 2323$-valent pneumococcal polysaccharides vaccine, $V T$ vaccine type

\section{Vaccine Impact on All-Type IPD in All Ages}

Data were available from 10 articles from Australia (two), western Europe (six) and North America (two) (Table 5). The prevaccination rate of all-type IPD per 100,000 varied from 4.0 in Germany [13] to 24.4 in the USA [33]. The postvaccination rate per 100,000 varied from 3.2 in Germany [13] to 17.1 in Denmark [12]. Eight studies reported a reduction in IPD incidence ranging from $9.6 \%$ in England [25] to $44.7 \%$ in the USA [33]; the median rate of reduction was $26.8 \%$. The highest impact was observed in the USA, Australia, and the UK. In the UK, a higher reduction rate was observed in the later post-PCV7 period (20092010 vs. 2003-2005). Two studies reported small, statistically nonsignificant increases; $3.2 \%$ in Spain [22] and 6.0\% in the Netherlands [14].

\section{Impact on VT IPD in Age Groups not Vaccine Eligible (Indirect Effect)}

Seven of the eight studies reporting data for nonvaccine-eligible children under 17 years 
of age showed a reduction in VT IPD ranging from $40.0 \%$ in children aged 5-17 years in Spain and Alaska, USA [23, 35] to $78.3 \%$ in children aged 5-14 years in Western Australia [44] (Appendix Table 1). One study reported an increase of $150.0 \%$ in children aged 5-17 years in a postvaccination period soon after the introduction of PCV7 [34]; in a later period, for the same population, a reduction of $40.0 \%$ was reported [35].

The impact on VT IPD in adults aged 15/1764 years was reported in 12 studies for a total of 18 age groups. A reduction was reported in all studies except one; in the Netherlands an increase of $9.9 \%$ was reported in those aged 50-64 years [14]. The reductions reported ranged

Table 5 Impact on all-type IPD in all ages

\begin{tabular}{|c|c|c|c|c|c|c|}
\hline \multirow{2}{*}{$\begin{array}{l}\text { Study (year of PCV7 } \\
\text { implementation) [ref] }\end{array}$} & \multirow{2}{*}{$\begin{array}{l}\text { Study } \\
\text { population }\end{array}$} & \multicolumn{2}{|c|}{ Prevaccination } & \multicolumn{2}{|c|}{ Postvaccination } & \multirow[t]{2}{*}{$\%$ change } \\
\hline & & Years & $\begin{array}{l}\text { Incidence/ } \\
100,000\end{array}$ & Years & $\begin{array}{l}\text { Incidence/ } \\
100,000\end{array}$ & \\
\hline \multicolumn{7}{|l|}{ Australia (January 2005 for all) } \\
\hline Lehmann 2010 [44] & $\begin{array}{l}\text { All ages, } \\
\text { nonaboriginal } \\
\text { people (PCV7 } \\
\text { \& PPV23) }\end{array}$ & $1997-2001$ & 7.5 & $2005-2007$ & 4.8 & -36.0 \\
\hline Hanna $2010[43]$ & $\begin{array}{l}\text { All ages, } \\
\text { nonaboriginal } \\
\text { people }\end{array}$ & $2001-2004$ & 9.3 & $2006-2009$ & 6.1 & -34.4 \\
\hline \multicolumn{7}{|l|}{ Europe } \\
\hline $\begin{array}{l}\text { Foster 2011, UK } \\
\text { (September 2006) [25] }\end{array}$ & All ages & $2003-2005$ & 8.3 & $2006-2009$ & 7.5 & -9.6 \\
\hline $\begin{array}{l}\text { Miller 2011, UK } \\
\text { (September 2006) [26] }\end{array}$ & All ages & $2000-2006$ & 16.1 & $2009-2010$ & 10.6 & -34.2 \\
\hline $\begin{array}{l}\text { Rückinger 2009, } \\
\text { Germany [13] }\end{array}$ & $\begin{array}{l}<16 \text { years } \\
\text { (capture- } \\
\text { recapture) }\end{array}$ & $1997-2003$ & 4.0 & $2007-2008$ & 3.2 & -20.0 \\
\hline Harboe 2010, Denmark [12] & All ages & $2000-2007$ & 19.6 & 2008 & 17.1 & -12.8 \\
\hline $\begin{array}{l}\text { Guevara 2009, Spain (June } \\
\text { 2001: limited) [22] }\end{array}$ & All ages & $2001-2002$ & 15.8 & $2006-2007$ & 16.3 & +3.2 \\
\hline $\begin{array}{l}\text { Rodenburg 2010, } \\
\text { Netherlands } \\
\text { (June 2006) [14] }\end{array}$ & All ages & 2004-2006 & 15.0 & $2006-2008$ & 15.9 & +6.0 \\
\hline North America & & & & & & \\
\hline $\begin{array}{l}\text { Pilishvili } 2010 \\
\text { (late 2000) [33] }\end{array}$ & All ages & $1998-1999$ & 24.4 & 2007 & 13.5 & -44.7 \\
\hline $\begin{array}{l}\text { Singleton } 2007 \\
\text { (January 2001) [35] }\end{array}$ & $\begin{array}{l}\text { All ages, } \\
\text { nonnatives }\end{array}$ & $1995-2000$ & 16.7 & $2004-2006$ & 11.1 & -33.5 \\
\hline
\end{tabular}

$I P D$ invasive pneumococcal disease, $P C V 77$-valent pneumococcal conjugate vaccine, $P P V 2323$-valent pneumococcal polysaccharides vaccine 
from $7.1 \%$ in those aged $30-49$ years in Western Australia [44] to $90.8 \%$ in those aged $18-49$ years in the USA [33].

All 13 studies reporting the impact for adults aged 65 years or older (with a total of 16 age groups) showed a reduction in the incidence of VT IPD ranging from $1.1 \%$ in the Netherlands [14] to $92.0 \%$ in the USA [33], with a median reduction of $53.8 \%$.

\section{Impact on All-Type IPD in Age Groups not Vaccine Eligible (Indirect Effect)}

There was an overall decrease for 12 of the 16 studies that reported data for nonvaccineeligible children aged from over 2 or over 5 years to less than 17 years; the rate reduction ranged from $6.6 \%$ for children aged 2 years or older in the UK [25] to $53.4 \%$ in children aged 2-4 years in Norway [15] (Appendix Table 2). In the five studies that reported an increase, the rate increase ranged from $9.1 \%$ in children aged 5-15 years in Germany [13] to 90.5\% and $134.5 \%$ in children aged 5-17 years and 2-4 years, respectively, in one hospital in Barcelona, Spain [23].

Fourteen studies reported data in 25 adult age groups (from 15/17 to 64 years old). The rates reported for 19 of these age groups ranged from a reduction of $2.0 \%$ in 15-64 year-old nonnatives in north Queensland, Australia [43] to $48.9 \%$ in 18-44 year-old nonnatives in Alaska, USA [34]. In the six age groups in which an increased rate was reported, the increase ranged from $8.5 \%$ in nonnative 50-64 year olds in north Queensland, Australia [43] to $119.7 \%$ in $20-39$ year olds in Alberta, Canada [41].

Seventeen studies reported data for those aged 65 years and over, with one study from Calgary, Canada reporting data for two age groups; 65-84 years and 85 years and over [42]. Reductions were reported in 12 of these studies, ranging from $7.1 \%$ in Denmark [12] to $44.1 \%$ in the USA [39]. In the remaining age groups, the increased rate reported ranged from $2.4 \%$ in the Netherlands [14] to $22.9 \%$ in Spain [17]. In one study from Canada, a reduction of $34.0 \%$ and an increase of $9.3 \%$ were reported for those aged 65-84 years and 85 year olds and older, respectively [42].

\section{Impact on All-Type IPD and VT IPD Mortality}

The impact of PCV7 vaccination on IPD mortality (both all-type [four studies] and VT [two studies]) was reported from North America only. One study reported reductions for VT IPD mortality across all age groups [33]. In another study, the reduction in VT IPD mortality in children less than 2 years observed was offset with an increase in non-VT IPD mortality resulting in no change [31]. Two studies reported reductions of $62.5 \%$ and $57.1 \%$ for alltype IPD in vaccine-eligible children [33, 38]. Two studies reported an overall reduction in all-type IPD mortality in all ages of $37.8 \%$ and $75.9 \%[38,40]$. There was a reduction in all-type IPD mortality for all the nonvaccine-eligible age groups reported ranging from $17.4 \%$ in those aged 50 years or older [30] to $50.0 \%$ in those aged 25-34 years [38].

\section{DISCUSSION}

The results from this review illustrate that PCV7 has had a significant impact on IPD across all ages through its use in pediatric immunization programs. PCV7 vaccination leads to a reduction in VT IPD in vaccine-eligible children and also to an overall reduction in all-type IPD in these children. PCV7 was also shown to have an indirect impact on VT and all-type IPD in the overall population, although this impact is 
lower than that seen in vaccine-eligible children. Despite the increase in non-VT IPD that has been reported, it has been insufficient generally to offset the reduction of VT IPD resulting in an overall reduction of all-type IPD.

The extent of the impact varied across the studies; many factors (and probably a combination of factors) contribute to explain this heterogeneity.

One of these factors is the sero-epidemiology of $S$. pneumoniae before the introduction of PCV7 and the match with the serotypes in PCV7. In the USA, the serotypes included in PCV7 matched the most prevalent serotypes responsible for IPD, but in other countries and regions, the percentage of IPD due to these seven serotypes varied; thus partly explaining the different impact observed [25, 32].

The process for PCV7 introduction, its uptake, the recommended schedule, and the implementation of a catch-up program or not also varied between countries. In the USA, where the impact was the greatest, there was a rapid and high uptake after introduction into the NIP for universal vaccination of children less than 2 years old with a $3+1$ schedule [47]. However, there was a vaccine shortage in the early stages after introduction into the NIP, so uptake of all four doses was low during this period [48]. In Australia, PCV7 was funded in mid-2001 for indigenous children less than 2 years of age, nonindigenous children living in central Australia, and for all children less than 5 years of age with predisposing medical conditions [44]. The recommended schedule was $3+0$ (at 2, 4, and 6 months old) with no booster in the second year of life. Then in January 2005, PCV7 funding was extended to all children less than 2 years of age.

In Europe, although PCV7 received its licence in 2001, introduction into NIPs and funding varied widely. In some countries, such as the UK, following the introduction of PCV7 and reimbursement for all children under 2 years of age in 2006 with a $2+1$ schedule, uptake was rapid and high, with an estimated uptake rate from $84.7 \%$ in 2007-2008 to 93\% in 2009-2010 in the UK [49]. In other countries, such as France and Switzerland, PCV7 was introduced into the NIPs with a 3 +1 schedule initially for at-risk children less than 2 years of age in France and under 5 years of age in Switzerland. In 2006, PCV7 was also recommended for all children under 2 years of age with a $2+1$ schedule in France, and in Switzerland for healthy children under 2 years of age. In France, the incidence of all-type IPD in children less than 2 years was reduced by 30\% between 2001-2002 and 2007 [50]. In Switzerland, the number of children under 2 years of age with all-type IPD was reduced from an average of 43 to 22 in 2009 (49\% reduction) [51]. For the same periods, the reduction in VT IPD was $85 \%$ (from 27 to 4 ) while the number of non-VT IPD cases remained stable [51].

The periods chosen for prevaccination and postvaccination can also introduce variability in the estimated impact. It is difficult to differentiate changes that can occur independently of vaccination, such as natural evolution of circulating serotypes or antibiotic resistance, from changes due to vaccineinduced serotype replacement, and it is possible that several factors may be responsible for the changes. Data from the USA show an evolution of impact with a greater distance from PCV7 introduction for the postvaccination period. In Alaska, comparing the incidence of VT IPD in nonnative children under 2 years of age in 1995-2000 with that in 2001-2003 and 2004-2006 showed reductions of $80.3 \%$ and $97.7 \%$, respectively (Table 2) [34, 35]. The same analysis for all-type IPD showed reductions of $61.7 \%$ and $67.8 \%$, respectively. In another 
USA dataset, the ABCs network, the analysis for 2001 just after the introduction of PCV7 showed a $78.5 \%$ reduction in the incidence of VT IPD in children lesst than 2 years of age compared with the analysis for 2005 , which showed almost $100 \%$ reduction $[29,32]$.

The studies analyzed in this review used different surveillance systems and case definitions; in addition, it is possible that changes in diagnosis and reporting practices occurred after the introduction of PCV7. The studies also varied in their geographical coverage, with some able to claim regional or national representation, while some reported data from one or two hospitals, which was the case for four studies from Spain [17, 20, 23, 24]. In addition, different schedules $(3+1$ and $2+1)$ were used with differing rates of uptake; for example, in Norway and the UK, there was a rapid uptake after introduction into the NIPs with a $2+1$ schedule, whereas in Spain there was a slow uptake after introduction through the private market with a $3+1$ schedule.

Despite these limitations, this analysis generally confirms the impact of PCV7 on VT IPD and all-type IPD in vaccine-eligible children as well as an indirect impact on nonvaccineeligible age groups. Since 2010 many countries have decided to replace PCV7 with PCV13. The vaccine includes the seven serotypes present in PCV7 (i.e., 14, 4, 9V, 19F, 18C, 6B, and 23F), and offers potential additional protection against serotypes 1, 5, 7F, 19A, 6A, and 3 . PCV13 has been shown to be well tolerated and immunogenic for all 13 serotypes [52]. Although no PCV13 impact study results have been published yet, in the UK where PCV13 replaced PCV7 in April 2010, preliminary data suggest that, up to July 2011, IPD due to PCV13-only serotypes has been reduced by $50 \%$ in children under 2 years of age [53].

\section{CONCLUSION}

It is important not to lose sight of the significant impact that PCV7 has had on IPD, despite the increase in non-VT IPD, but we need to remain vigilant for serotype replacement. With the introduction of PCV13 we can expect to see a reduction in the incidence of IPD due to the six additional serotypes included, as well as continued protection against IPD due to PCV7 serotypes. Robust surveillance systems are essential for the evaluation of the impact of PCV13 on all-type IPD and for monitoring the evolution of non-VT IPD.

\section{ACKNOWLEDGMENTS}

EpiConcept performed the literature search, study selection and data extraction; this work was funded by Pfizer. Pfizer also paid the article publication charges. The authors take full responsibility for the interpretation and discussion of the data. Editorial assistance in the preparation of this manuscript was provided by Dr. Margaret Haugh, MediCom Consult; this was funded by Pfizer. Dr. Myint is the guarantor for this article, and takes responsibility for the integrity of the work as a whole.

Conflict of interest. Dr. Myint is employed by Pfizer, the manufacturer of Prevenar ${ }^{\circledR}$ and Prevenar $13^{\circledR}$, Wyeth Pharmaceuticals Inc, marketed by Pfizer Inc, New York, USA. Dr. Madhava, Dr. Balmer, Dr. Christopoulou, Dr. Attal, Dr. Menegas, and Dr. Bonnet are all employed by Pfizer. Dr. Sprenger is employed by Pfizer at the time of submission.

Open Access. This article is distributed under the terms of the Creative Commons Attribution Noncommercial License, which permits any non-commercial use, distribution, and reproduction in any medium, provided the original author(s) and source are credited. 


\section{APPENDIX}

Table 1 Impact on VT IPD reduction in nonvaccine-eligible populations

\begin{tabular}{|c|c|c|c|c|c|c|c|}
\hline & \multirow[t]{2}{*}{ Study $[$ ref $]$} & \multirow[t]{2}{*}{ Study population } & \multicolumn{2}{|c|}{ Prevaccination } & \multicolumn{2}{|c|}{ Postvaccination } & \multirow[t]{2}{*}{$\%$ change } \\
\hline & & & Years & $\begin{array}{l}\text { Incidence/ } \\
100,000\end{array}$ & Years & $\begin{array}{l}\text { Incidence/ } \\
100,000\end{array}$ & \\
\hline \multirow{16}{*}{ 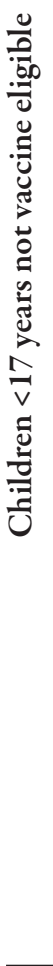 } & Australia & & & & & & \\
\hline & Hanna $2010[43]$ & Nonaboriginal 5-14 years & $2001-2004$ & 1.2 & 2006-2009 & 0.6 & -50.0 \\
\hline & Lehmann 2010 [44] & Nonaboriginal $2-4$ years & $1997-2001$ & 17.9 & $2005-2007$ & 4.2 & -76.5 \\
\hline & & Nonaboriginal $5-14$ years & & 2.3 & & 0.5 & -78.3 \\
\hline & Europe & & & & & & \\
\hline & Miller 2011, UK [26] & $5-14$ years & $2000-2006$ & 2.2 & $2009-2010$ & 0.5 & -77.3 \\
\hline & $\begin{array}{l}\text { Vestrheim 2008, } \\
\text { Norway [15] }\end{array}$ & $2-4$ years & 2004-2005 & 13.5 & 2007 & 7.5 & -44.4 \\
\hline & Munoz-Almagro 2008, & $2-4$ years & $1997-2001$ & 6.8 & $2002-2006$ & 9.2 & +35.3 \\
\hline & Spain [23] & $5-17$ years & & 0.5 & & 0.3 & -40.0 \\
\hline & North America & & & & & & \\
\hline & Hennessy 2005 [34] & Nonnative $2-4$ years & $1995-2000$ & 13.6 & $2001-2003$ & 7.5 & -44.9 \\
\hline & & Nonnative $5-17$ years & & 1.0 & & 2.5 & +150.0 \\
\hline & Singleton 2007 [35] & Nonnative 2-4 years & $1995-2000$ & 13.6 & $2004-2006$ & 0 & -100.0 \\
\hline & & Nonnative $5-17$ years & & 1.0 & & 0.6 & -40.0 \\
\hline & Kellner 2009 [42] & $2-4$ years & $1998-2001$ & 17.9 & $2003-2007$ & 7.3 & -59.2 \\
\hline & & $5-15$ years & & 2.5 & & 1.3 & -48.0 \\
\hline \multirow{12}{*}{ 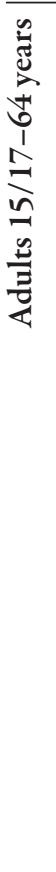 } & Australia & & & & & & \\
\hline & Hanna 2010 [43] & $15-64$ years & 2001-2004 & 3.1 & 2006-2009 & 1.2 & -61.3 \\
\hline & Lehmann 2010 [44] & $\begin{array}{l}\text { Nonaboriginal 15-29 } \\
\text { years }\end{array}$ & $1997-2001$ & 1.6 & $2005-2007$ & 0.7 & -56.3 \\
\hline & & $\begin{array}{l}\text { Nonaboriginal 30-49 } \\
\text { years }\end{array}$ & & 1.4 & & 1.3 & -7.1 \\
\hline & & $\begin{array}{l}\text { Nonaboriginal 50-64 } \\
\text { years }\end{array}$ & & 3.4 & & 2.8 & -17.7 \\
\hline & Europe & & & & & & \\
\hline & Miller 2011, UK [26] & $15-44$ years & $2000-2006$ & 3.3 & 2009-2010 & 0.4 & -87.9 \\
\hline & & $45-64$ years & & 7.7 & & 1.1 & -85.7 \\
\hline & Vestrheim 2010, & 20-39 years & 2004-2005 & 3.9 & 2008 & 3.1 & -20.5 \\
\hline & Norway [16] & $40-64$ years & & 12.2 & & 4.9 & -59.8 \\
\hline & Ardanuy 2009, Spain [17] & $18-64$ years & $1997-2001$ & 3.1 & $2005-2007$ & 2.7 & -12.9 \\
\hline & $\begin{array}{l}\text { Rodenburg 2010, } \\
\text { Netherlands [14] }\end{array}$ & $50-64$ years & $2004-2006$ & 7.5 & $2006-2008$ & 8.2 & +9.3 \\
\hline
\end{tabular}


Table 1 continued

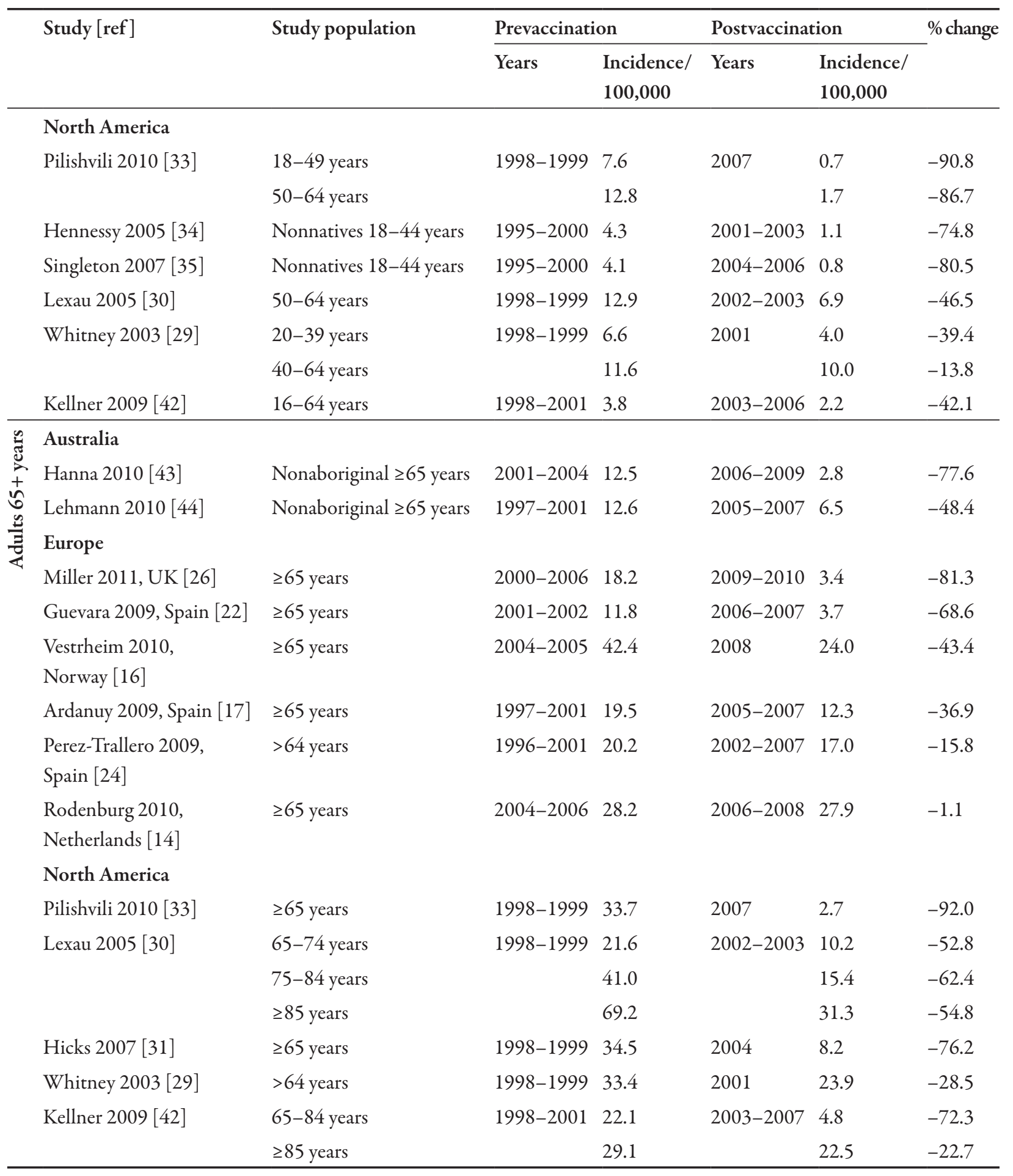

$V T I P D$ vaccine-type invasive pneumococcal disease 
Table 2 Impact on all-type IPD in nonvaccine-eligible populations

\begin{tabular}{|c|c|c|c|c|c|c|c|}
\hline & \multirow[t]{2}{*}{ Study $[$ ref $]$} & \multirow[t]{2}{*}{ Study population } & \multicolumn{2}{|c|}{ Prevaccination } & \multicolumn{2}{|c|}{ Postvaccination } & \multirow[t]{2}{*}{$\%$ chang } \\
\hline & & & Years & $\begin{array}{l}\text { Incidence/ } \\
100,000\end{array}$ & Years & $\begin{array}{l}\text { Incidence/ } \\
100,000\end{array}$ & \\
\hline \multirow{24}{*}{ 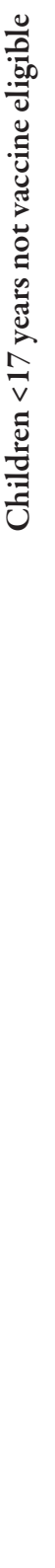 } & Australia & & & & & & \\
\hline & Hanna $2010[43]$ & Nonaboriginal $5-14$ years & 2001-2004 & 2.5 & 2006-2009 & 1.2 & -52.0 \\
\hline & Lehmann 2010 [44] & Nonaboriginal 2-4 years & $1997-2001$ & 21.2 & $2005-2007$ & 9.9 & -53.3 \\
\hline & & Nonaboriginal $5-14$ years & & 2.8 & & 1.7 & -39.3 \\
\hline & Europe & & & & & & \\
\hline & $\begin{array}{l}\text { Verstrheim 2008, } \\
\text { Norway [15] }\end{array}$ & $2-4$ years & 2004-2005 & 23.6 & 2007 & 11.0 & -53.4 \\
\hline & Miller 2011, UK [26] & $2-4$ years & $2000-2006$ & 16.4 & $2009-2010$ & 9.3 & -43.3 \\
\hline & & $5-14$ years & & 5.1 & & 3.0 & -41.2 \\
\hline & Aristegui 2007, Spain [18] & $2-5$ years & $1998-2001$ & 30.1 & $2002-2003$ & 33.1 & -10.0 \\
\hline & Foster 2011, UK [25] & $\geq 2$ years & $2003-2005$ & 7.6 & 2006-2009 & 7.1 & -6.6 \\
\hline & $\begin{array}{l}\text { Rückinger 2009, } \\
\text { Germany [13] }\end{array}$ & $5-15$ years & $1997-2003$ & 1.1 & $2007-2008$ & 1.2 & +9.1 \\
\hline & Harboe 2010, & $2-4$ years & $2000-2007$ & 8.5 & 2008 & 11.8 & +38.8 \\
\hline & Denmark [12] & $5-17$ years & & 2.6 & & 1.5 & -42.3 \\
\hline & Munoz-Almagro 2008, & $2-4$ years & $1997-2001$ & 11.3 & $2002-2006$ & 26.5 & +134.5 \\
\hline & Spain $[23]$ & $5-17$ years & & 2.1 & & 4.0 & +90.5 \\
\hline & North America & & & & & & \\
\hline & Pilishvili 2010 [33] & $5-17$ years & $1998-1999$ & 4.2 & 2007 & 2.4 & -42.9 \\
\hline & Hennessy 2005 [34] & Nonnatives $5-17$ years & $1995-2000$ & 3.9 & 2004-2006 & 4.6 & +17.9 \\
\hline & Singleton 2007 [35] & Nonnatives $5-17$ years & $1995-2000$ & 3.8 & $2001-2003$ & 2.8 & -26.3 \\
\hline & Albrich 2007 [27] & $5-17$ years & $1997-2000$ & 3.9 & 2000-2004 & 3.3 & -15.4 \\
\hline & Tyrrell 2009 [41] & $5-19$ years & 2000 & 2.4 & 2006 & 4.0 & +66.7 \\
\hline & Kellner 2009 [42] & $2-4$ years & $1998-2001$ & 22.7 & 2003-2007 & 12.6 & -44.5 \\
\hline & & $5-15$ years & & 3.3 & & 2.4 & -27.3 \\
\hline & Simonsen 2011 [39] & $5-17$ years & $1996-1999$ & 1.4 & $2005-2006$ & 0.7 & -50.0 \\
\hline \multirow{5}{*}{ 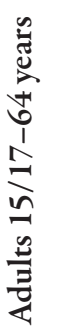 } & Australia & & & & & & \\
\hline & Lehmann 2010 [44] & Nonaboriginal $15-29$ years & $1997-2001$ & 2.4 & $2005-2007$ & 1.5 & -37.5 \\
\hline & & Nonaboriginal $30-49$ years & & 2.8 & & 2.7 & -3.6 \\
\hline & Hanna $2010[43]$ & Nonaboriginal 50-64 years & $2001-2004$ & 4.7 & $2006-2009$ & 5.1 & +8.5 \\
\hline & & Nonaboriginal $15-64$ years & & 5.0 & & 4.9 & -2.0 \\
\hline
\end{tabular}


Table 2 continued

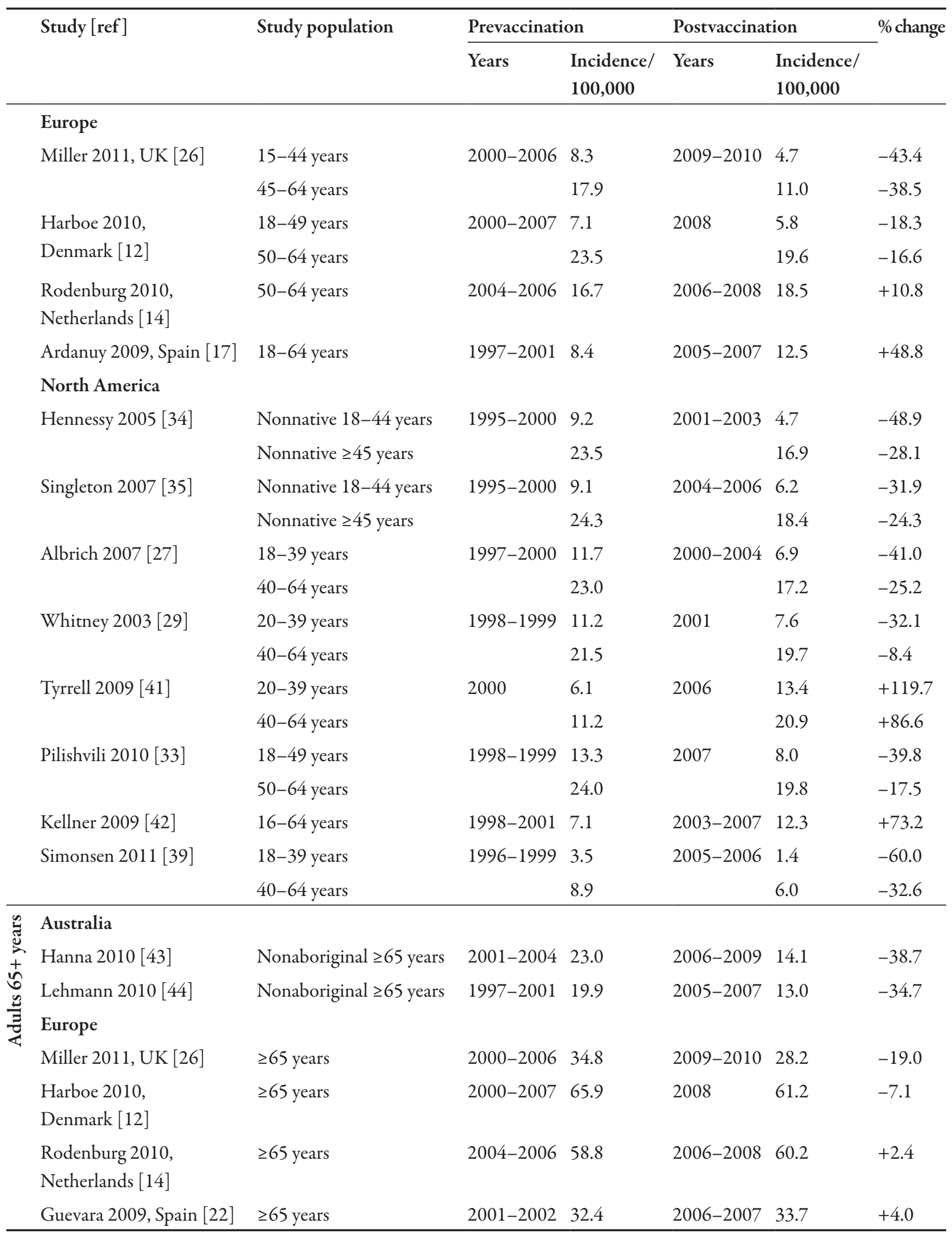


Table 2 continued

\begin{tabular}{|c|c|c|c|c|c|c|}
\hline \multirow[t]{2}{*}{ Study [ref] } & \multirow[t]{2}{*}{ Study population } & \multicolumn{2}{|c|}{ Prevaccination } & \multicolumn{2}{|c|}{ Postvaccination } & \multirow[t]{2}{*}{$\%$ change } \\
\hline & & Years & $\begin{array}{l}\text { Incidence/ } \\
100,000\end{array}$ & Years & $\begin{array}{l}\text { Incidence/ } \\
100,000\end{array}$ & \\
\hline $\begin{array}{l}\text { Perez-Trallero 2009, } \\
\text { Spain [24] }\end{array}$ & $>64$ years & $1996-2001$ & 50.7 & $2002-2007$ & 55.6 & +9.7 \\
\hline Ardanuy 2009, Spain [17] & $\geq 65$ years & $1997-2001$ & 45.8 & $2005-2007$ & 56.3 & +22.9 \\
\hline \multicolumn{7}{|l|}{ North America } \\
\hline McBean 2005 [37] & $\geq 65$ years & $1996-2000$ & 36.8 & $2002-2003$ & 21.8 & -40.8 \\
\hline Hicks 2007 [31] & $\geq 65$ years & $1998-1999$ & 61.5 & 2004 & 38.0 & -38.2 \\
\hline Pilishvili 2010 [33] & $\geq 65$ years & $1998-1999$ & 60.1 & 2007 & 37.9 & -36.9 \\
\hline Lexau 2005 [30] & $\geq 50$ years & 1998-1999 & 40.8 & $2002-2003$ & 29.4 & -27.9 \\
\hline Albrich 2007 [27] & $\geq 65$ years & $1997-2000$ & 65.4 & $2000-2004$ & 48.2 & -26.3 \\
\hline Whitney 2003 [29] & $\geq 65$ years & $1998-1999$ & 60.1 & 2001 & 49.5 & -17.6 \\
\hline Tyrrell 2009 [41] & $\geq 65$ years & 2000 & 28.2 & 2006 & 23.0 & -18.4 \\
\hline \multirow[t]{2}{*}{ Kellner 2009 [42] } & $65-84$ years & $1998-2001$ & 36.2 & $2003-2007$ & 23.9 & -34.0 \\
\hline & $\geq 85$ years & & 55.0 & & 60.1 & +9.3 \\
\hline Simonsen 2011 [39] & $\geq 65$ years & $1996-1999$ & 30.6 & $2005-2006$ & 17.1 & -44.1 \\
\hline
\end{tabular}

$I P D$ invasive pneumococcal disease

\section{REFERENCES}

1. Johnson HL, Deloria-Knoll M, Levine OS, et al. Systematic evaluation of serotypes causing invasive pneumococcal disease among children under five: the pneumococcal global serotype project. PLoS Med. 2010;7:e1000348.

2. Frenck RW Jr, Yeh S. The development of 13 -valent pneumococcal conjugate vaccine and its possible use in adults. Expert Opin Biol Ther. 2012;12:63-77.

3. O'Brien KL, Wolfson LJ, Watt JP, et al. Burden of disease caused by Streptococcus pneumoniae in children younger than 5 years: global estimates. Lancet. 2009;374:893-902.

4. Pebody RG, Hellenbrand W, D'Ancona F, Ruutu P. Pneumococcal disease surveillance in Europe. Euro Surveill. 2006;11:171-8.

5. Rosen JB, Thomas AR, Lexau CA, et al. CDC Emerging Infectious Program Network. Geographic variation in invasive pneumococcal disease following pneumococcal conjugate vaccine introduction in the United States. Clin Infect Dis. 2011;53:137-43.
6. Fitzwater SP, Chandran A, Santosham M, Johnson HL. The worldwide impact of the seven-valent pneumococcal conjugate vaccine. Pediatr Infect Dis J. 2012;31:501-8.

7. Rose M, Zielen S. Impact of infant immunization programs with pneumococcal conjugate vaccine in Europe. Expert Rev Vaccines. 2009;8:1351-64.

8. Zangeneh TT, Baracco G, Al-Tawfiq JA. Impact of conjugate pneumococcal vaccines on the changing epidemiology of pneumococcal infections. Expert Rev Vaccines. 2011;10:345-53.

9. World Health Organization. Pneumococcal conjugate vaccine for childhood immunization - WHO position paper. Wkly Epidemiol Rec. 2007;82:93-104.

10. Rendi-Wagner P, Paulke-Korinek M, Kundi M, et al. National paediatric immunization program of high risk groups: no effect on the incidence of invasive pneumococcal diseases. Vaccine. 2009;27:3963-8.

11. Hanquet $G$, Lernout $T$, Vergison $A$, et al. Belgian IPD Scientific Committee. Impact of conjugate 7-valent vaccination in Belgium: addressing methodological challenges. Vaccine. 2011;29:2856-64. 
12. Harboe ZB, Valentiner-Branth P, Benfield TL, et al. Early effectiveness of heptavalent conjugate pneumococcal vaccination on invasive pneumococcal disease after the introduction in the Danish Childhood Immunization Programme. Vaccine. 2010;28:2642-7.

13. Rückinger $S$, van der Linden $M$, Reinert RR, von Kries R, Burckhardt F, Siedler A. Reduction in the incidence of invasive pneumococcal disease after general vaccination with 7 -valent pneumococcal conjugate vaccine in Germany. Vaccine. 2009;27:4136-41.

14. Rodenburg GD, de Greeff SC, Jansen AG, et al. Effects of pneumococcal conjugate vaccine 2 years after its introduction, the Netherlands. Emerg Infect Dis. 2010;16:816-23.

15. Vestrheim DF, Lovoll O, Aaberge IS, et al. Effectiveness of a $2+1$ dose schedule pneumococcal conjugate vaccination programme on invasive pneumococcal disease among children in Norway. Vaccine. 2008;26:3277-81.

16. Vestrheim DF, Hoiby EA, Bergsaker MR, Ronning $\mathrm{K}$, Aaberge IS, Caugant DA. Indirect effect of conjugate pneumococcal vaccination in a $2+1$ dose schedule. Vaccine. 2010;28:2214-21.

17. Ardanuy C, Tubau F, Pallares R, et al. Epidemiology of invasive pneumococcal disease among adult patients in barcelona before and after pediatric 7-valent pneumococcal conjugate vaccine introduction, 1997-2007. Clin Infect Dis. 2009;48:57-64.

18. Aristegui J, Bernaola E, Pocheville I, et al. Reduction in pediatric invasive pneumococcal disease in the Basque Country and Navarre, Spain, after introduction of the heptavalent pneumococcal conjugate vaccine. Eur J Clin Microbiol Infect Dis. 2007;26:303-10.

19. Barricarte A, Gil-Setas A, Torroba L, et al. Invasive pneumococcal disease in children younger than 5 years in Navarra, Spain (2000-2005). Impact of the conjugate vaccine [in Spanish]. Med Clin (Barc). 2007;129:41-5.

20. Calbo E, Diaz A, Canadell E, et al. Invasive pneumococcal disease among children in a health district of Barcelona: early impact of pneumococcal conjugate vaccine. Clin Microbiol Infect. 2006;12:867-72.

21. Fenoll A, Granizo JJ, Aguilar L, et al. Temporal trends of invasive Streptococcus pneumoniae serotypes and antimicrobial resistance patterns in Spain from 1979 to 2007. J Clin Microbiol. 2009;47:1012-20.
22. Guevara M, Barricarte A, Gil-Setas A, et al. Changing epidemiology of invasive pneumococcal disease following increased coverage with the heptavalent conjugate vaccine in Navarre, Spain. Clin Microbiol Infect. 2009;15:1013-19.

23. Munoz-Almagro C, Jordan I, Gene A, Latorre C, Garcia-Garcia JJ, Pallares R. Emergence of invasive pneumococcal disease caused by nonvaccine serotypes in the era of 7-valent conjugate vaccine. Clin Infect Dis. 2008;46:174-82.

24. Perez-Trallero E, Marimon JM, Ercibengoa M, Vicente D, Perez-Yarza EG. Invasive Streptococcus pneumoniae infections in children and older adults in the north of Spain before and after the introduction of the heptavalent pneumococcal conjugate vaccine. Eur J Clin Microbiol Infect Dis. 2009;28:731-8.

25. Foster D, Walker AS, Paul J, et al. Oxford Invasive Penumococcal Surveillance Group. Reduction in invasive pneumococcal disease following implementation of the conjugate vaccine in the Oxfordshire region, England. J Med Microbiol. 2011;60:91-7.

26. Miller E, Andrews NJ, Waight PA, Slack MP, George RC. Herd immunity and serotype replacement 4 years after seven-valent pneumococcal conjugate vaccination in England and Wales: an observational cohort study. Lancet Infect Dis. 2011;11:760-8.

27. Albrich WC, Baughman W, Schmotzer B, Farley MM. Changing characteristics of invasive pneumococcal disease in Metropolitan Atlanta, Georgia, after introduction of a 7-valent pneumococcal conjugate vaccine. Clin Infect Dis. 2007;44:1569-76.

28. Black S, France EK, Isaacman D, et al. Surveillance for invasive pneumococcal disease during 20002005 in a population of children who received 7-valent pneumococcal conjugate vaccine. Pediatr Infect Dis J. 2007;26:771-7.

29. Whitney CG, Farley MM, Hadler J, et al. Active Bacterial Core Surveillance of the Emerging Infectious Program Network. Decline in invasive pneumococcal disease after the introduction of protein-polysaccharide conjugate vaccine. N Engl J Med. 2003;348:1737-46.

30. Lexau CA, Lynfield R, Danila R, et al. Active Bacterial Core Surveillance Team. Changing epidemiology of invasive pneumococcal disease among older adults in the era of pediatric pneumococcal conjugate vaccine. JAMA. 2005;294:2043-51. 
31. Hicks LA, Harrison LH, Flannery B, et al. Incidence of pneumococcal disease due to nonpneumococcal conjugate vaccine (PCV7) serotypes in the United States during the era of widespread PCV7 vaccination, 1998-2004. J Infect Dis. 2007;196:1346-54.

32. Centers for Disease Control and Prevention. Invasive pneumococcal disease in children 5 years after conjugate vaccine introduction - eight states, 1998-2005. MMWR Morb Mortal Wkly Rep. 2008;57:144-8.

33. Pilishvili T, Lexau C, Farley MM, et al. Active Bacterial Core Surveillance/Emerging Infectious Program. Sustained reductions in invasive pneumococcal disease in the era of conjugate vaccine. J Infect Dis. 2010;201:32-41.

34. Hennessy TW, Singleton RJ, Bulkow LR, et al. Impact of heptavalent pneumococcal conjugate vaccine on invasive disease, antimicrobial resistance and colonization in Alaska Natives: progress towards elimination of a health disparity. Vaccine. 2005;23:5464-73.

35. Singleton RJ, Hennessy TW, Bulkow LR, et al. Invasive pneumococcal disease caused by nonvaccine serotypes among alaska native children with high levels of 7-valent pneumococcal conjugate vaccine coverage. JAMA. 2007;297:1784-92.

36. Hsu K, Pelton S, Karumuri S, Heisey-Grove D, Klein J. Massachusetts Department of Public Health Epidemiologists. Population-based surveillance for childhood invasive pneumococcal disease in the era of conjugate vaccine. Pediatr Infect Dis J. $2005 ; 24: 17-23$.

37. McBean AM, Park YT, Caldwell D, Yu X. Declining invasive pneumococcal disease in the US elderly. Vaccine. 2005;23:5641-5.

38. Pulido M, Sorvillo F. Declining invasive pneumococcal disease mortality in the United States, 1990-2005. Vaccine. 2010;28:889-92.

39. Simonsen L, Taylor RJ, Young-Xu Y, Haber M, May L, Klugman KP. Impact of pneumococcal conjugate vaccination of infants on pneumonia and influenza hospitalization and mortality in all age groups in the United States. mBio. 2011;2:e00309-10.

40. Tsigrelis C, Tleyjeh IM, Lahr BD, Nyre LM, Virk A, Baddour LM. Decreases in case-fatality and mortality rates for invasive pneumococcal disease in Olmsted County, Minnesota, during 19952007: a population-based study. Clin Infect Dis. 2008;47:1367-71.
41. Tyrrell GJ, Lovgren M, Chui N, et al. Serotypes and antimicrobial susceptibilities of invasive Streptococcus pneumoniae pre- and postseven valent pneumococcal conjugate vaccine introduction in Alberta, Canada, 2000-2006. Vaccine. 2009;27:3553-60.

42. Kellner JD, Vanderkooi OG, MacDonald J, Church DL, Tyrrell GJ, Scheifele DW. Changing epidemiology of invasive pneumococcal disease in Canada, 1998-2007: update from the Calgary-area Streptococcus pneumoniae Research (CASPER) study. Clin Infect Dis. 2009;49:205-12.

43. Hanna JN, Humphreys JL, Murphy DM, Smith HV. Invasive pneumococcal disease in non-indigenous people in north Queensland, 2001-2009. Med J Aust. 2010;193:392-6.

44. Lehmann D, Willis J, Moore HC, et al. The changing epidemiology of invasive pneumococcal disease in aboriginal and non-aboriginal western Australians from 1997 through 2007 and emergence of nonvaccine serotypes. Clin Infect Dis. 2010;50:1477-86.

45. Roche PW, Krause V, Cook H, et al. Pneumococcal Working Party of the Communicable Disease Network Australia. Invasive pneumococcal disease in Australia, 2006. Commun Dis Intell. 2008;32:18-30.

46. Williams SR, Mernagh PJ, Lee MH, Tan JT. Changing epidemiology of invasive pneumococcal disease in Australian children after introduction of a 7-valent pneumococcal conjugate vaccine. Med J Aust. 2011;194:116-20.

47. Centers for Disease Control and Prevention. National, state, and urban area vaccination coverage among children aged 19-35 months United States, 2004. MMWR Morb Mortal Wkly Rep. 2005;54:717-21.

48. Broder KR, MacNeil A, Malone S, et al. Who's calling the shots? Pediatricians' adherence to the 2001-2003 pneumococcal conjugate vaccine-shortage recommendations. Pediatrics. 2005;115:1479-87.

49. HM Government. data.gov.uk. NHS Information Centre for Health and Social Care. NHS Immunisation Statistics England 2009-10. London: 2010. Available at: http://data.gov.uk/dataset/nhsimmunisation-statistics-england-2009-10. Accessed Jan 212013. 
50. Lepoutre A, Varon E, Georges S, Gutmann L, Levy-Bruhl D. Impact of pneumococcal conjugate vaccine on the epidemiology of invasive pneumococcal diseases in France - 1998-2010 [in French]. 2010 [cited Jun 14 2012]. Available at: http://www.invs.sante.fr/surveillance/epibac/ donnees_2010/pneumocoque_impact_2010.pdf. Accessed Jun 142012.

51. Office federal de la sante publique et Commission federale pour les vaccinations. Pneumococcal vaccination recommendations for children under five years old. Replacement of 7-valent conjugate vaccine by 13-valent conjugate vaccine [in French]. 2010 [cited Nov 9 2012];
Available from: http://www.bag.admin.ch/ themen/medizin/00682/00684/01097/index.html? lang=fr\#sprungmarke1_2. Accessed Nov 92012.

52. Esposito S, Tansey S, Thompson A, et al. Safety and immunogenicity of a 13-valent pneumococcal conjugate vaccine compared to those of a 7-valent pneumococcal conjugate vaccine given as a three-dose series with routine vaccines in healthy infants and toddlers. Clin Vaccine Immunol. 2010;17:1017-26.

53. Miller E, Andrews NJ, Waight PA, Slack MP, George RC. Effectiveness of the new serotypes in the 13-valent pneumococcal conjugate vaccine. Vaccine. 2011;29:9127-31. 